\title{
Reheating-induced axion dark matter after low scale inflation
}

\author{
Takeshi Kobayashi $^{a}$ and Lorenzo Ubaldi ${ }^{b, c}$ \\ ${ }^{a}$ Kobayashi-Maskawa Institute for the Origin of Particles and the Universe, \\ Nagoya University, Nagoya 464-8602, Japan \\ ${ }^{b}$ SISSA and INFN Sezione di Trieste, \\ Via Bonomea 265, 34136 Trieste, Italy \\ ${ }^{c}$ Institute for Fundamental Physics of the Universe, \\ Via Beirut 2, 34014 Trieste, Italy \\ E-mail: takeshi@kmi.nagoya-u.ac.jp, ubaldi.physics@gmail.com
}

\begin{abstract}
A kinetic mixing between the axion and the inflaton allows for a production of axion dark matter even if the inflationary Hubble scale is smaller than the zero-temperature axion mass. We analyze the axion dynamics in this recently discovered "inflaxion" framework, and present a new cosmological scenario where the axion drifts away from its vacuum during the reheating epoch, giving rise to the observed dark matter abundance. We discuss the implications for both the QCD axion and axion-like particles.
\end{abstract}

KEywords: Cosmology of Theories beyond the SM, Effective Field Theories

ARXiv EPrint: 2006.09389 


\section{Contents}

1 Introduction 1

2 Vacuum misalignment scenario $\quad 3$

3 Inflaxion scenario $\quad 6$

$\begin{array}{ll}3.1 \text { Basic setup } & 6\end{array}$

$\begin{array}{ll}3.2 & \text { End of inflation and reheating }\end{array}$

$\begin{array}{ll}3.3 \text { Drifting away from the vacuum } & 10\end{array}$

$\begin{array}{lll}3.4 & \text { Numerical examples } & 12\end{array}$

4 Parameter space $\quad 15$

4.1 Case study: $\sigma F \tilde{F}+\phi \bar{\psi} i \gamma^{5} \psi \quad 16$

$\begin{array}{lll}4.2 & \text { Upper bound on inflaton mass } & 18\end{array}$

$\begin{array}{lll}4.3 & \text { QCD inflaxion } & 18\end{array}$

$\begin{array}{llr}5 & \text { Conclusions } & 20\end{array}$

$\begin{array}{ll}\text { A Onset of axion oscillation } & 21\end{array}$

$\begin{array}{ll}\text { B Diagonal basis } & 23\end{array}$

\section{Introduction}

Most of the matter in the Universe is dark, but we have yet to discover what it is composed of. A plausible candidate is an axion, a spin zero boson which appears in many models of particle physics. The best motivated one is the QCD axion [1,2], which arises as a solution to the strong CP problem [3]. String theory compactifications also provide motivation to contemplate axions, whose masses and couplings span many orders of magnitude [4, 5]. There is a growing experimental effort aimed at covering much of this parameter space, with the hope of detecting a dark matter axion. On the other hand, there is still room on the theoretical side to explore new production mechanisms for axion dark matter, that can open up new regions of parameters, and provide guidance for the experiments. The aim of this work is to present a new production scenario, by building up and expanding on an idea we have recently put forward [6].

An axion is a pseudo Nambu-Goldstone Boson of a spontaneously broken U(1) global symmetry (which is the Peccei-Quinn symmetry [3] for the QCD axion), and is characterized by the scale of symmetry breaking $f$. If the $\mathrm{U}(1)$ is also explicitly broken by a coupling with a strong gauge group, as is the case for the QCD axion, then the confinement scale $\Lambda$ is another important quantity. Given a hierarchy between the two scales $\Lambda \ll f$, the 
zero-temperature axion mass $m \sim \Lambda^{2} / f$ is suppressed. In textbook treatments and in a large part of the literature it is usually assumed that the Hubble scale of inflation is high compared to the axion mass,

$$
H_{\text {inf }} \gg m \text {. }
$$

Then, an important distinction is whether the maximum value between $H_{\text {inf }}$ and the largest temperature $T_{\max }$ reached in the Universe, is larger or smaller than $f$. If it is larger, $\max .\left\{H_{\mathrm{inf}}, T_{\max }\right\} \gg f$, the Universe goes through a phase transition from unbroken to broken $\mathrm{U}(1)$ after inflation. This gives rise to topological defects including axionic strings, which later emit axions and give a contribution to the dark matter relic density, although the actual amount produced is still uncertain [7,8]. If instead max. $\left\{H_{\text {inf }}, T_{\max }\right\} \ll f$, the $\mathrm{U}(1)$ is broken during and after inflation. Then the main source of axion dark matter production is the random initial displacement of the axion field from the vacuum, and this is often referred to as the vacuum misalignment scenario [9-11].

However, we still do not know the scale of inflation. In fact, it could be as low as

$$
H_{\text {inf }}<m,
$$

as long as there is enough energy density available to reheat the Universe to a temperature above $\mathrm{MeV}$ where Big Bang Nucleosynthesis (BBN) takes place. With such a low $H_{\text {inf }}$, the axion is considered to undergo damped oscillations during inflation and settles at the minimum of its potential, reaching the point of zero energy density and thus contributing nothing to the dark matter abundance. This logic, however, neglects possible couplings between the axion and the inflaton sector. If they do not violate the axion shift symmetry, there is no a priori reason to forbid direct interactions between the two scalars from the point of view of an effective field theory.

The cosmological consequences of an inflaton-axion coupling was explored in ref. [6], where we demonstrated that a dimension-four kinetic mixing can lead to a production of axion dark matter even with a low scale inflation of (1.2). ${ }^{1}$ The mechanism proceeds as follows. The axion during inflation is stabilized close to the bottom of its potential, but is kicked out at the end of inflation due to the kinetic coupling as the inflaton rapidly rolls towards its vacuum. This process displaces the axion field from the vacuum and sources axion dark matter. The inflaton-axion system can also be studied in the field basis where the kinetic and mass terms are diagonalized. If the inflaton mass at the vacuum is larger than the axion mass, then a consistent post-inflation cosmological history can be realized where the heavier of the two diagonal fields decays and reheats the universe, while the lighter one survives and serves as dark matter. The reheaton and dark matter fields are both linear combinations of the inflaton and axion, hence are dubbed the inflaxions.

In the study of the inflaxion mechanism in ref. [6], we primarily focused on cases where the axion potential stays constant during the cosmic evolution. However the potential can also vary in time if it arises from a coupling with a strong gauge group, as is the case for the QCD axion. In this work we explore the possibility that after low scale inflation, the cosmic temperature during the reheating phase exceeds the confinement scale $\Lambda$, and hence

\footnotetext{
${ }^{1}$ For studies of kinetic mixings among multiple axions, see e.g. [12-16].
} 
the axion potential temporarily vanishes. This gives rise to rich dynamics of the inflatonaxion system, allowing for a new cosmological scenario for axion dark matter. Here, the reheating phase plays a central role in the dark matter production, and we show how this opens up new regions of the axion parameter space. We study the implications for both the QCD axion, and axion-like particles coupled to a hidden confining gauge sector.

This paper is organized as follows: We start by reviewing the conventional vacuum misalignment scenario in section 2. Then we discuss the inflaxion mechanism in section 3 , followed by a study of its parameter space in section 4 . We then conclude in section 5 . Technical calculations are relegated to the appendices: The onset of the axion oscillation is analyzed in detail in appendix A. The full expressions for the diagonal basis of the inflaxion Lagrangian are listed in appendix B.

\section{Vacuum misalignment scenario}

Let us start by reviewing the conventional vacuum misalignment scenario. Throughout this work we denote the axion by $\sigma$, and consider it to be coupled to some gauge force that becomes strong in the IR at an energy scale $\Lambda$. We assume the axion mass to depend on the cosmic temperature $T$ as

$$
m_{\sigma}(T) \simeq \begin{cases}\lambda m_{\sigma 0}\left(\frac{\Lambda}{T}\right)^{p} & \text { for } T \gg \Lambda, \\ m_{\sigma 0} & \text { for } T \ll \Lambda,\end{cases}
$$

with the zero-temperature mass written as

$$
m_{\sigma 0}=\xi \frac{\Lambda^{2}}{f} .
$$

Here $\xi$ is a dimensionless parameter, and $f$ is an axion decay constant which sets the periodicity of the axion potential as $\sigma \cong \sigma+2 \pi f$. For the QCD axion, the parameters take the values $\Lambda \approx 200 \mathrm{MeV}, p \approx 4, \lambda \approx 0.1, \xi \approx 0.1$, and $f$ is the only free parameter. However, in order to keep the discussion general, we take all the parameters as arbitrary positive numbers.

The vacuum misalignment scenario can work if the inflationary Hubble scale lies within the range

$$
m_{\sigma}\left(T_{\text {inf }}\right)<H_{\text {inf }}<2 \pi f
$$

where $T_{\mathrm{inf}}=H_{\mathrm{inf}} / 2 \pi$ is the de Sitter temperature during inflation. The upper bound indicates that the $\mathrm{U}(1)$ symmetry is already broken ${ }^{2}$ in the inflation epoch and thus the axion field becomes homogeneous in the observable patch of the universe. The cosmic temperature during reheating should also satisfy the same upper bound, $T<f$, to ensure that the symmetry stays broken in the post-inflation universe. The lower bound on the inflation scale indicates that any initial field displacement $\sigma_{\star}$ of the axion from its potential minimum stays frozen during inflation due to the Hubble friction. The axion continues to

\footnotetext{
${ }^{2}$ The symmetry breaking scale can be different from the scale of the axion periodicity, however we suppose the two scales to be of the same order throughout this paper.
} 
stay frozen in the post-inflationary universe while $m_{\sigma}<H$, but eventually starts to oscillate about its potential minimum when the Hubble scale becomes as small as $m_{\sigma}>H$. The oscillating field corresponds to a collection of axion particles with a high occupation number and very small momentum. The particle number is then approximately conserved, and the physical number density can be written as

$$
n_{\sigma} \simeq \frac{1}{2} m_{\sigma}\left(T_{\mathrm{osc}}\right) \sigma_{\star}^{2}\left(\frac{a_{\mathrm{osc}}}{a}\right)^{3} \quad \text { for } \quad m_{\sigma} \gg H,
$$

in terms of quantities at the onset of the field oscillation which are denoted by the subscript (osc). Given that the axion starts to oscillate during radiation domination ${ }^{3}$ at temperatures $T \gg \Lambda$ (i.e. when $m_{\sigma} \propto T^{-p}$ ), then the 'onset' of the oscillation can be defined as when the ratio between the axion mass and the Hubble scale becomes

$$
\frac{m_{\sigma}\left(T_{\mathrm{osc}}\right)}{H_{\mathrm{osc}}}=(2 p+4)\left[\pi^{-\frac{1}{2}} \Gamma\left(\frac{2 p+5}{2 p+4}\right)\right]^{\frac{2 p+4}{p+3}} \equiv c_{p} .
$$

This definition of the onset renders the expression (2.5) for the number density exact in the asymptotic future $a \rightarrow \infty$, as shown in appendix A ( $c_{p}$ corresponds to (A.9) with the substitution $w=1 / 3)$. For instance, the QCD axion with $p \approx 4$ exhibits $c_{4} \approx 4$. The Hubble scale during radiation domination is related to the cosmic temperature via $3 M_{\mathrm{Pl}}^{2} H^{2} \simeq \rho_{\mathrm{r}}=\left(\pi^{2} / 30\right) g_{*}(T) T^{4}$, which can be combined with (2.1) and (2.6) to give the temperature at the onset of the oscillation as ${ }^{4}$

$$
T_{\mathrm{osc}} \simeq \Lambda\left\{\left(\frac{\pi^{2}}{90} g_{*}\left(T_{\mathrm{osc}}\right)\right)^{-1 / 2} \frac{\lambda \xi}{c_{p}} \frac{M_{\mathrm{Pl}}}{f}\right\}^{\frac{1}{p+2}} .
$$

Considering the entropy of the universe to be conserved since the onset of the oscillation, the entropy density $s=\left(2 \pi^{2} / 45\right) g_{s *}(T) T^{3} \propto a^{-3}$ can be used to express the axion's number density in the current universe as

$$
n_{\sigma 0}=\frac{1}{2} m_{\sigma}\left(T_{\mathrm{osc}}\right) \sigma_{\star}^{2} \frac{s_{0}}{s_{\mathrm{osc}}},
$$

where the subscript 0 represents quantities today. Supposing $T_{0} \ll \Lambda$, the present-day axion density is $\rho_{\sigma 0}=m_{\sigma 0} n_{\sigma 0}$, and thus by combining with the equations above one can obtain the density parameter as

$$
\begin{aligned}
\Omega_{\sigma} h^{2}=\kappa_{p} \theta_{\star}^{2}\left(\frac{g_{s *}\left(T_{\mathrm{osc}}\right)}{100}\right)^{-1}\left(\frac{g_{*}\left(T_{\mathrm{osc}}\right)}{100}\right)^{\frac{p+3}{2 p+4}}\left(\frac{\lambda}{0.1}\right)^{-\frac{1}{p+2}}\left(\frac{\xi}{0.1}\right)^{\frac{p+1}{p+2}} \\
\times\left(\frac{\Lambda}{200 \mathrm{MeV}}\right)\left(\frac{f}{10^{12} \mathrm{GeV}}\right)^{\frac{p+3}{p+2}} .
\end{aligned}
$$

Here $\theta_{\star} \equiv \sigma_{\star} / f$ is the initial misalignment angle. $\kappa_{p}$ is a numerical factor that depends on the power $p$, whose value is plotted in the left panel of figure 1 ; for instance, $\kappa_{4} \approx 0.1$. Combinations of $f$ and $\Lambda$ that yield the observed dark matter abundance, $\Omega_{\sigma} h^{2} \approx 0.1$ [20], are shown in the right panel of figure 1.

\footnotetext{
${ }^{3}$ For discussions on cases where radiation domination takes over after the onset of the oscillations, see $[17-19]$.

${ }^{4}$ Throughout this paper $M_{\mathrm{Pl}}$ refers to the reduced Planck mass $(8 \pi G)^{-1 / 2}$.
} 

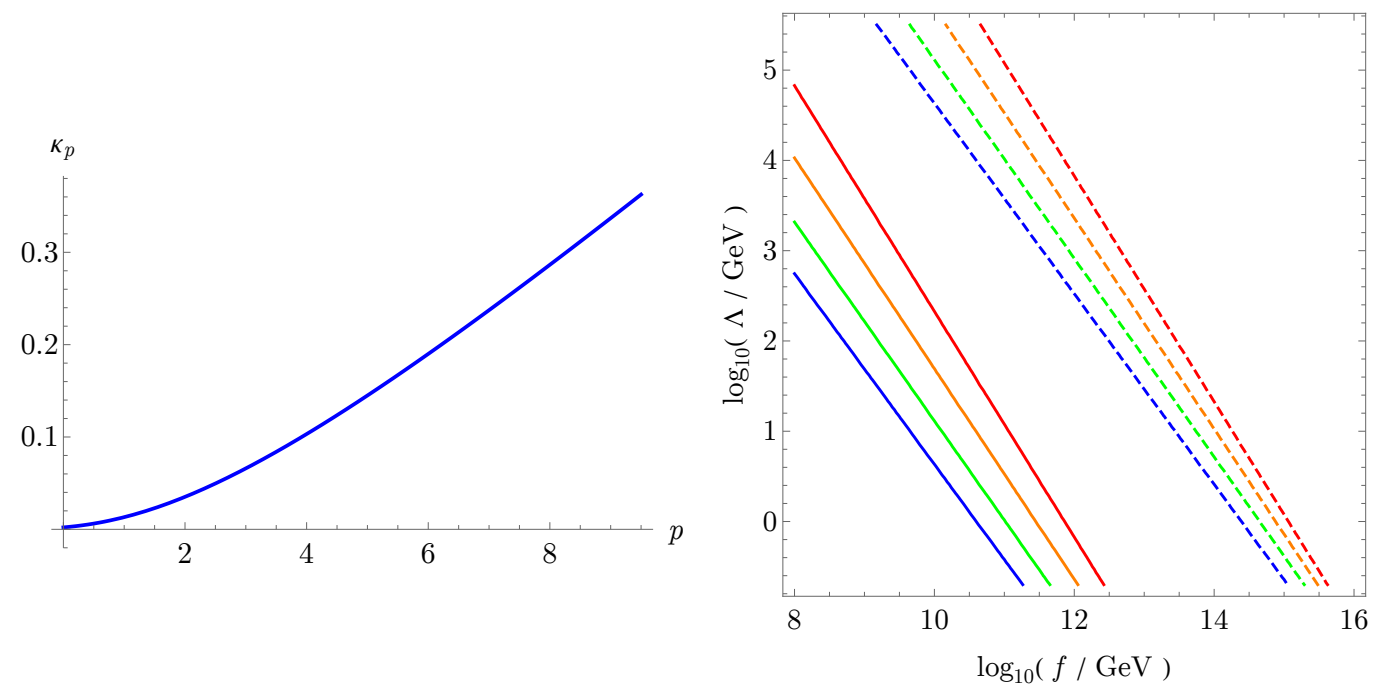

Figure 1. Left: Value of the numerical factor $\kappa_{p}$ for the axion abundance (2.9), as a function of the power $p$ of the temperature dependence. Right: Contours in the plane of the axion decay constant $f$ and strong coupling scale $\Lambda$ that give rise to the observed dark matter abundance from a vacuum misalignment, for values of the power $p=2$ (red), 4 (orange), 8 (green), 16 (blue), and misalignment angle $\theta_{\star}=1$ (solid lines), $10^{-2}$ (dashed lines). Other parameters are fixed to $\lambda=0.1$, $\xi=0.1, g_{*}\left(T_{\mathrm{osc}}\right)=g_{s *}\left(T_{\mathrm{osc}}\right)=100$. The lower edge of the plot represents $\Lambda=200 \mathrm{MeV}$.

By taking the $p \rightarrow 0$ limit (which entails $\kappa_{p \rightarrow 0} \approx 0.002$ ) along with $\lambda \rightarrow 1,(2.9)$ reduces to the relic abundance of an axion with a constant mass $m_{\sigma 0}$ (cf., e.g., eq. (3.10) of [21]). On the other hand, as $\kappa_{p}$ is a monotonically increasing function of $p$, the abundance (2.9) is enhanced for a large $p$. However it should also be noted that upon deriving this result, the axion was assumed to start oscillating while its mass varies with the temperature as $m_{\sigma} \propto T^{-p}$. This amounts to assuming that the axion mass at the onset of the oscillation is smaller than the zero-temperature mass, i.e. $\lambda\left(\Lambda / T_{\text {osc }}\right)^{p}<1$, which combined with $(2.7)$ translates into an upper bound on the decay constant,

$$
f<\left(\frac{\pi^{2}}{90} g_{*}\left(T_{\mathrm{osc}}\right)\right)^{-1 / 2} c_{p}^{-1} \lambda^{-\frac{2}{p}} \xi M_{\mathrm{Pl}} .
$$

This condition is satisfied for all values of $f$ plotted in the right panel of figure 1 . One can also check that the condition breaks down at large values of $p$, and hence, of course, the relic abundance actually does not increase indefinitely with $p$.

We should also remark that we have ignored self-interactions of the axion. Since the axion potential is periodic as $\sigma \cong \sigma+2 \pi f$, the relic abundance would receive anharmonic corrections when the misalignment angle is as large as $\left|\theta_{\star}\right| \gtrsim 1[22,23]$.

Finally, we note that when the axion makes up a significant fraction of the dark matter in our universe, the upper limit of the inflation scale window (2.4) for the vacuum misalignment scenario becomes much more restrictive due to observational constraints on dark matter isocurvature perturbations (see e.g. [24]). 


\section{$3 \quad$ Inflaxion scenario}

\subsection{Basic setup}

It was discovered in [6] that even when the inflation scale is as low as

$$
H_{\text {inf }}<m_{\sigma}\left(T_{\text {inf }}\right),
$$

axion dark matter can be produced by invoking a kinetic mixing between the axion and the inflaton. ${ }^{5}$ The basic idea is captured by the following theory:

$$
\frac{\mathcal{L}}{\sqrt{-g}}=-\frac{1}{2} g^{\mu \nu} \partial_{\mu} \sigma \partial_{\nu} \sigma-\frac{1}{2} m_{\sigma}(T)^{2} \sigma^{2}-\frac{1}{2} g^{\mu \nu} \partial_{\mu} \phi \partial_{\nu} \phi-V(\phi)-\alpha g^{\mu \nu} \partial_{\mu} \phi \partial_{\nu} \sigma+L_{\mathrm{c}}[\sigma, \phi, \Psi] .
$$

Here, $\sigma$ is the axion whose mass term is understood to arise from expanding the periodic potential around one of the minima, $\phi$ is the inflaton with a potential $V(\phi)$ that possesses an inflationary plateau, $\alpha$ is a dimensionless coupling constant that satisfies $|\alpha|<1$ to avoid ghost degrees of freedom, and $L_{\mathrm{c}}$ represents couplings with other matter fields which we collectively denote by $\Psi$. Given that the axion is a pseudoscalar, the inflaton would also need to be a pseudoscalar for the kinetic mixing term to conserve parity; however we remark that parity conservation is not a prerequisite for the mechanism to operate.

The main part of the analysis in [6] was devoted to axions with a constant mass; for axions coupled to a strong sector, this amounts to assuming that the cosmic temperature never exceeds the strong coupling scale $\Lambda$. In the following, we instead analyze the case where the temperature in the post-inflation universe becomes higher than $\Lambda$, but lower than $f$, so that the axion mass temporarily diminishes, while the $\mathrm{U}(1)$ symmetry continues to be broken. To be concrete, we consider the axion mass to depend on the temperature as (2.1) and (2.2), and focus on cases where the inflationary de Sitter temperature $T_{\mathrm{inf}}$ and the maximum temperature of radiation $T_{\max }$ during the reheating process satisfy

$$
T_{\mathrm{inf}}<\Lambda<T_{\max }<f .
$$

As in [6], the inflationary Hubble scale is considered to be smaller than the zero-temperature axion mass, which in turn is smaller than the inflaton mass $m_{\phi 0}$ at the vacuum. In the following we further assume that the axion mass becomes smaller than the Hubble scale when the temperature reaches $T_{\max }{ }^{6}$ Thus we impose the following hierarchy:

$$
m_{\sigma}\left(T_{\max }\right)<H_{\mathrm{inf}}<m_{\sigma 0}<m_{\phi 0} .
$$

The time evolution of the temperature and the scalar field masses are illustrated in figure 2. Here, $a_{\text {end }}$ represents the scale factor when inflation ends. In the left panel,

\footnotetext{
${ }^{5}$ Variants of this mechanism can also be constructed with a potential coupling that respects the axion's discrete shift symmetry, or a coupling of the axion to other fields such as the waterfall field in hybrid inflation [25].

${ }^{6}$ We have in mind here perturbative reheating in which $T_{\max }$ is reached within about a Hubble time after the end of inflation. Hence the Hubble rate upon $T=T_{\max }$ is of the same order as the Hubble rate at the end of inflation.
} 

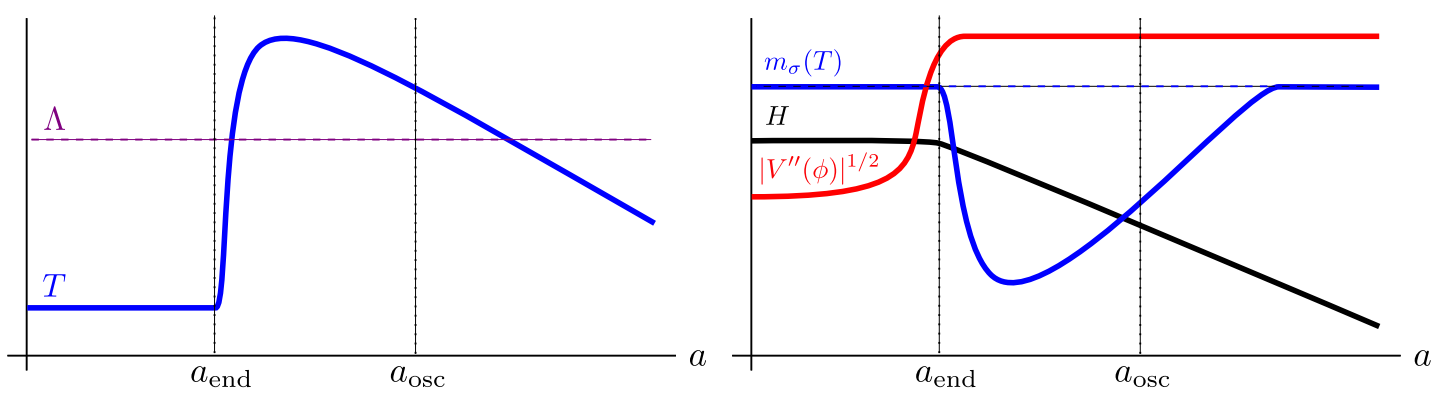

Figure 2. Schematic of the time evolution of the cosmic temperature (left) and scalar field masses (right) in the inflaxion scenario under consideration (not to scale).

the cosmic temperature during inflation is taken as the de Sitter temperature, while after inflation it is the radiation temperature arising from the decay of the reheaton. (The de Sitter and radiation temperatures are connected at the end of inflation for illustration purposes only.) The right panel shows the evolution of the Hubble rate $H$ (black curve), the axion mass $m_{\sigma}(T)$ (blue), and the effective mass $\left|V^{\prime \prime}(\phi)\right|^{1 / 2}$ of the inflaton (red). The axion mass during inflation takes its zero-temperature value $m_{\sigma 0}$, then during reheating becomes smaller than $H$ for a while, and again becomes $m_{\sigma 0}$ in the later universe when $T \ll \Lambda$. The inflaton potential $V(\phi)$ is considered to possess a plateau that enables slowroll inflation, and hence $\left|V^{\prime \prime}(\phi)\right|^{1 / 2}<H$ during inflation. This inequality breaks down towards the end of inflation, as the inflaton rolls to its vacuum. Inflation thus ends and the inflaton starts to oscillate around the minimum of its potential, which we assume to be approximated by a quadratic,

$$
V(\phi) \simeq \frac{1}{2} m_{\phi 0}^{2} \phi^{2} \quad \text { for } \quad|\phi| \leq\left|\phi_{\text {end }}\right| .
$$

Here, $\phi_{\text {end }}$ refers to the inflaton field value where inflation ends. The inflaton mass thus becomes $m_{\phi 0}$, which is larger than $m_{\sigma 0}$ as required in (3.4). We have in mind here smallfield inflation models in which $\left|V^{\prime \prime}(\phi)\right|^{1 / 2}$ takes very different values between the plateau region and the minimum. However we should also note that this transition of $\left|V^{\prime \prime}(\phi)\right|^{1 / 2}$ from a tiny value during inflation to a larger $m_{\phi 0}$ is not necessarily monotonic as shown in the simplified illustration; $\left|V^{\prime \prime}(\phi)\right|^{1 / 2}$ can instead oscillate due to higher order terms in the potential while the inflaton's oscillation amplitude is large.

\subsection{End of inflation and reheating}

The post-inflationary dynamics of the inflaton and axion is insensitive to the details of the inflation model, and thus we start our discussion from the time when inflation ends.

Let us for a moment ignore the temperature dependence of the axion mass. Then one can simultaneously diagonalize the kinetic terms as well as the mass terms so that the Lagrangian (3.2) with the quadratic inflaton potential (3.5) is rewritten as

$$
\frac{\mathcal{L}}{\sqrt{-g}}=\sum_{i=\mathrm{DM}, \mathrm{RH}}\left(-\frac{1}{2} g^{\mu \nu} \partial_{\mu} \varphi_{i} \partial_{\nu} \varphi_{i}-\frac{1}{2} m_{i}^{2} \varphi_{i}^{2}\right)+L_{\mathrm{c}}[\sigma, \phi, \Psi]
$$


where the explicit forms of the diagonalized fields and their masses are given in appendix B. Here, we suppose a mass hierarchy $m_{\sigma}^{2} \ll m_{\phi 0}^{2}$ and use approximate expressions of

$$
\begin{array}{rlrl}
\varphi_{\mathrm{DM}} & \simeq \alpha \phi+\sigma, & \varphi_{\mathrm{RH}} & \simeq \sqrt{1-\alpha^{2}}\left(\phi-\alpha \frac{m_{\sigma}^{2}}{m_{\phi 0}^{2}} \sigma\right), \\
m_{\mathrm{DM}} \simeq m_{\sigma}, & m_{\mathrm{RH}} \simeq \frac{m_{\phi 0}}{\sqrt{1-\alpha^{2}}},
\end{array}
$$

where each of the coefficients of $\phi$ and $\sigma$, as well as the diagonalized masses are given to leading order in a $m_{\sigma}^{2} / m_{\phi 0}^{2}$ expansion. ( $\varphi_{\mathrm{DM}}$ and $\varphi_{\mathrm{RH}}$ correspond respectively to $\varphi_{+}$and $\varphi_{-}$in (B.3) up to overall signs.) Recalling $\alpha^{2}<1$, one sees that $m_{\mathrm{DM}} \simeq m_{\sigma}<m_{\phi 0}<m_{\mathrm{RH}}$. The lighter field $\varphi_{\text {DM }}$ can be long-lived for a sufficiently small axion mass, and thus serves as a dark matter candidate. The heavier field $\varphi_{\mathrm{RH}}$, on the other hand, can reheat the universe through its decay.

The diagonal basis is also convenient for analyzing the decay of the scalar particles. If, for instance, the axion and inflaton were coupled to (either Standard Model (SM) or hidden) photons and/or a light Dirac fermion $\psi$ via

$$
L_{\mathrm{c}}[\sigma, \phi, \Psi]=\frac{G_{\sigma \gamma \gamma}}{4} \sigma F_{\mu \nu} \tilde{F}^{\mu \nu}+\frac{G_{\phi \gamma \gamma}}{4} \phi F_{\mu \nu} \tilde{F}^{\mu \nu}+g_{\phi f f} \phi \bar{\psi} i \gamma^{5} \psi
$$

then the decay widths of the dark matter and reheaton are given to leading order in $m_{\sigma}^{2} / m_{\phi 0}^{2}$ as (see also (B.5) and (B.6) for the full expressions)

$$
\begin{aligned}
\Gamma\left(\varphi_{\mathrm{DM}} \rightarrow \gamma \gamma\right) \simeq \frac{G_{\sigma \gamma \gamma}^{2}}{64 \pi} m_{\sigma}^{3}, & \Gamma\left(\varphi_{\mathrm{RH}} \rightarrow \gamma \gamma\right) \simeq \frac{\alpha^{2}}{\left(1-\alpha^{2}\right)^{5 / 2}} \frac{G_{\sigma \gamma \gamma}^{2}}{64 \pi} m_{\phi 0}^{3}, \\
\Gamma\left(\varphi_{\mathrm{DM}} \rightarrow \gamma \gamma\right) \simeq \alpha^{2} \frac{G_{\phi \gamma \gamma}^{2}}{64 \pi} \frac{m_{\sigma}^{7}}{m_{\phi 0}^{4}}, & \Gamma\left(\varphi_{\mathrm{RH}} \rightarrow \gamma \gamma\right) \simeq \frac{1}{\left(1-\alpha^{2}\right)^{5 / 2}} \frac{G_{\phi \gamma \gamma}^{2}}{64 \pi} m_{\phi 0}^{3}, \\
\Gamma\left(\varphi_{\mathrm{DM}} \rightarrow f \bar{f}\right) \simeq \alpha^{2} \frac{g_{\phi f f}^{2}}{8 \pi} \frac{m_{\sigma}^{5}}{m_{\phi 0}^{4}}, & \Gamma\left(\varphi_{\mathrm{RH}} \rightarrow f \bar{f}\right) \simeq \frac{1}{\left(1-\alpha^{2}\right)^{3 / 2}} \frac{g_{\phi f f}^{2}}{8 \pi} m_{\phi 0} .
\end{aligned}
$$

The decay widths in each line are induced by each of the terms in (3.8), and here we have ignored the contribution to the two-photon decay rates from the cross-term $\propto G_{\sigma \gamma \gamma} G_{\phi \gamma \gamma}$. These expressions explicitly show that the life time of the reheaton is suppressed compared to that of the dark matter field by powers of their mass ratio.

Hereafter we promote the axion mass in the expressions of (3.7) to a temperaturedependent mass $m_{\sigma}(T)$, and analyze the post-inflationary dynamics in terms of the (pseudo)diagonal fields. Later on, we compare the results that follow from this analytic procedure with those obtained by solving the full set of equations of motion.

The inflaton field value at the end of inflation can be estimated by noting that a significant fraction of the total energy density of the universe is still in the inflaton's potential energy (3.5), i.e.,

$$
M_{\mathrm{Pl}}^{2} H_{\mathrm{end}}^{2} \sim m_{\phi 0}^{2} \phi_{\mathrm{end}}^{2},
$$

where we use the subscript (end) to represent quantities at the end of inflation. It was found in [6] that the axion field value becomes comparable to $\alpha \phi$ towards the end of inflation, 
and hence the dark matter field value is obtained as

$$
\varphi_{\mathrm{DM} \text { end }}^{2}=\left(\frac{C \alpha M_{\mathrm{Pl}} H_{\mathrm{end}}}{m_{\phi 0}}\right)^{2} \text {. }
$$

Here, $C$ is a numerical factor whose exact value depends on the model, and is typically of $C \sim 10$. The dark matter field begins to oscillate with this initial amplitude, with a mass equal to the zero-temperature axion mass, i.e. $m_{\mathrm{DM} \text { end }} \simeq m_{\sigma 0}$, since the reheaton $\varphi_{\mathrm{RH}}$ still has not started to decay at this point. The dark matter field's potential energy is

$$
\left(\frac{1}{2} m_{\mathrm{DM}}^{2} \varphi_{\mathrm{DM}}^{2}\right)_{\mathrm{end}} \simeq \frac{C^{2} \alpha^{2}}{6} \frac{m_{\sigma 0}^{2}}{m_{\phi 0}^{2}} \times 3 M_{\mathrm{Pl}}^{2} H_{\mathrm{end}}^{2},
$$

which is suppressed compared to the total energy density of the universe by the mass ratio $m_{\sigma 0}^{2} / m_{\phi 0}^{2}$. This indicates that the post-inflation universe is initially dominated by the reheaton.

The reheaton undergoes oscillations and decays into hot radiation, which forces the dark matter mass to diminish. We now evaluate the reheating process without specifying the explicit forms of the matter couplings. Here we only assume that reheating proceeds by a perturbative decay of the reheaton into radiation with a decay width $\Gamma_{\mathrm{RH}}$.

If $\Gamma_{\mathrm{RH}}<H_{\mathrm{end}}$, then the radiation density would reach its maximum value

$$
\rho_{\mathrm{r} \max } \sim \frac{\Gamma_{\mathrm{RH}}}{H_{\mathrm{end}}} M_{\mathrm{Pl}}^{2} H_{\mathrm{end}}^{2},
$$

within about a Hubble time after the end of inflation [26]. ${ }^{7}$ Subsequently the radiation density turns to redshift, albeit slowly as it continues to be sourced by the decaying reheaton, and eventually dominates over the reheaton density when $H \sim \Gamma_{\mathrm{RH}}$.

If on the other hand $\Gamma_{\mathrm{RH}}>H_{\text {end }}$, then the reheaton would quickly decay ${ }^{8}$ and radiation domination would take over right after the end of inflation, yielding

$$
\rho_{\mathrm{r} \max } \sim M_{\mathrm{Pl}}^{2} H_{\mathrm{end}}^{2} .
$$

${ }^{7}$ This can be checked explicitly by solving the continuity equation for the radiation density,

$$
\dot{\rho}_{\mathrm{r}}+4 H \rho_{\mathrm{r}}=\Gamma_{\mathrm{RH}} \rho_{\mathrm{RH}},
$$

with an initial condition $\rho_{\mathrm{r} \text { end }}=0$. Here, the energy density of the decaying reheaton can be written as

$$
\rho_{\mathrm{RH}}=\left(\frac{a}{a_{\text {end }}}\right)^{-3} e^{-\Gamma_{\mathrm{RH}}\left(t-t_{\text {end }}\right)} \rho_{\mathrm{RH} \text { end }},
$$

and considering the post-inflation universe to be initially dominated by the non-relativistic reheaton particles ( $\rho_{\mathrm{RH} \text { end }} \simeq 3 M_{\mathrm{Pl}}^{2} H_{\mathrm{end}}^{2}$ ) gives a scaling $H^{2} \propto a^{-3}$. Then the solution of the continuity equation, to linear order in $\Gamma_{\mathrm{RH}}$, is

$$
\rho_{\mathrm{r}} \simeq \frac{6}{5} \frac{\Gamma_{\mathrm{RH}}}{H_{\mathrm{end}}} M_{\mathrm{P} 1}^{2} H_{\mathrm{end}}^{2}\left\{\left(\frac{a}{a_{\mathrm{end}}}\right)^{-3 / 2}-\left(\frac{a}{a_{\text {end }}}\right)^{-4}\right\} \quad \text { for } \quad \Gamma_{\mathrm{RH}} \ll H .
$$

At $a_{\max } \approx 1.5 \times a_{\text {end }}$, this expression takes its maximum value

$$
\rho_{\mathrm{r} \max } \approx 0.4 \times \frac{\Gamma_{\mathrm{RH}}}{H_{\mathrm{end}}} M_{\mathrm{Pl}}^{2} H_{\mathrm{end}}^{2} .
$$

${ }^{8} \Gamma_{\mathrm{RH}}>H_{\text {end }}$ after inflation does not mean that the inflaton fluctuations should have decayed during inflation, since the effective mass of the inflaton during slow-roll inflation is much smaller than $m_{\phi 0}$. 
Thus for all cases $\Gamma_{\mathrm{RH}} \gtreqless H_{\text {end }}$, the Hubble rate when radiation domination takes over can be collectively written as

$$
H_{\mathrm{dom}} \sim \min .\left\{H_{\mathrm{end}}, \Gamma_{\mathrm{RH}}\right\}
$$

and the maximum radiation density as

$$
\rho_{\mathrm{r} \max } \sim \frac{H_{\mathrm{dom}}}{H_{\mathrm{end}}} M_{\mathrm{Pl}}^{2} H_{\mathrm{end}}^{2} .
$$

Therefore the maximum temperature during reheating is written as

$$
T_{\max } \sim 10^{4} \mathrm{GeV}\left(\frac{g_{*}\left(T_{\max }\right)}{100}\right)^{-1 / 4}\left(\frac{H_{\mathrm{end}}}{1 \mathrm{eV}}\right)^{1 / 2}\left(\frac{H_{\mathrm{dom}}}{H_{\mathrm{end}}}\right)^{1 / 4} .
$$

\subsection{Drifting away from the vacuum}

As the radiation temperature increases after the end of inflation, the axion mass becomes smaller than the Hubble scale. Then the dark matter field, being effectively massless, streams freely with the velocity that it had acquired before its mass diminished. In this way the field obtains a further displacement from its potential minimum. ${ }^{9}$

To make a rough estimate of this effect, note that at the end of inflation when the radiation temperature is effectively zero, ${ }^{10}$ the dark matter field is beginning to oscillate with the zero-temperature axion mass $m_{\mathrm{DM} \text { end }} \simeq m_{\sigma 0}$. The field velocity at this time is thus estimated as

$$
\left|\dot{\varphi}_{\text {DM end }}\right| \sim m_{\sigma 0}\left|\varphi_{\text {DM end }}\right|,
$$

where an overdot denotes a derivative with respect to physical time. Then if the temperature rises rapidly and hence the axion mass, or equivalently the dark matter mass, immediately vanishes, the dark matter field would begin to free-stream with the above initial velocity. However the Hubble friction damps the velocity of a free field, and so the dark matter field comes to a halt after a few Hubble times. ${ }^{11}$ Hence the field moves a distance of

$$
\left|\Delta \varphi_{\mathrm{DM}}\right| \sim\left|\frac{\dot{\varphi}_{\mathrm{DM}} \text { end }}{H_{\text {end }}}\right| \sim \frac{m_{\sigma 0}}{H_{\text {end }}}\left|\varphi_{\text {DM end }}\right|>\left|\varphi_{\text {DM end }}\right|,
$$

where we used (3.21) in the second approximation, and (3.4) for the last inequality. This indicates that the field excursion during the free-streaming dominates over the field displacement at the end of inflation. Hence by combining (3.23) with (3.13), the dark matter

\footnotetext{
${ }^{9}$ If instead the radiation temperature never exceeds $\Lambda$ and the axion mass stays constant, then the field displacement (3.13) at the very end of inflation would be the only source for dark matter production [6].

${ }^{10}$ Here perturbative reheating after inflation is assumed. However the radiation temperature may rise already before the inflaton begins to oscillate, if, for instance, tachyonic preheating [27] takes place. It would be interesting to explore the inflaxion mechanism in such cases as well.

${ }^{11}$ The velocity of a massless homogeneous field redshifts as $\dot{\varphi}_{\mathrm{DM}} \propto a^{-3}$. Integrating this from the end of inflation in a universe with a constant equation of state $w(\neq 1)$ yields
}

$$
\varphi_{\mathrm{DM}}-\varphi_{\mathrm{DM} \text { end }}=\frac{2}{3(1-w)} \frac{\dot{\varphi}_{\mathrm{DM} \text { end }}}{H_{\text {end }}}\left\{1-\left(\frac{a}{a_{\text {end }}}\right)^{-\frac{3(1-w)}{2}}\right\} .
$$


field value after the free-streaming is obtained as

$$
\varphi_{\mathrm{DM} \star}^{2}=\left(\frac{B \alpha M_{\mathrm{Pl}} m_{\sigma 0}}{m_{\phi 0}}\right)^{2} .
$$

Here, $B$ is a dimensionless parameter which is of order unity according to the discussions above. ${ }^{12}$ However we should also note that this is only a crude approximation and the actual field dynamics can be more intricate. For instance, if $m_{\sigma 0}$ is only marginally larger than $H_{\text {end }}$, then the free-streaming distance $\Delta \varphi_{\text {DM }}$ and the initial displacement $\varphi_{\text {DM end }}$ would be comparable in size and thus might cancel each other, yielding a much smaller field displacement. The approximation that the field begins to free-stream with the initial velocity (3.21) could also break down, if the time scale $\Delta t$ for the axion mass to diminish is larger than the initial oscillation period, i.e. $\Delta t \gtrsim 2 \pi / m_{\sigma 0}$; cases with a gradually decreasing mass will be discussed in detail in the next subsection where we numerically study concrete examples. All such effects that give corrections to the simplest picture discussed above would amount to shifting the parameter $B$ from order unity.

After the free-streaming, the dark matter field stays frozen at $\varphi_{\mathrm{DM} \star}$, and then begins to oscillate about its potential minimum as the cosmic temperature decreases and the axion mass again becomes larger than the Hubble rate. The field dynamics hereafter is the same as in the conventional vacuum misalignment scenario. In this sense, the temperaturedependent inflaxion can be considered as a mechanism that sources a vacuum misalignment of (3.24) with low scale inflation. Hence we can apply the results of section 2 to compute the dark matter abundance: Assuming radiation domination to take over before the dark matter field starts to oscillate, i.e.

$$
H_{\text {dom }}>H_{\mathrm{osc}},
$$

and also the axion mass to be varying with temperature at the onset of the oscillation, i.e. (2.10), then the present-day dark matter abundance can be computed as (2.9), with the misalignment angle given by

$$
\theta_{\star}^{2}=\left(\frac{\varphi_{\mathrm{DM} \star}}{f}\right)^{2}=\left(\frac{B \alpha M_{\mathrm{Pl}} m_{\sigma 0}}{f m_{\phi 0}}\right)^{2} .
$$

It is worth stressing that, unlike in the conventional vacuum misalignment scenario where the angle is given as a random initial condition, here it is uniquely fixed by the inflaxion parameters. We also note that the angle in this inflaxion scenario is independent of the inflation scale.

By the time the dark matter field starts to oscillate, the reheaton has decayed away and thus the field value of the inflaton is much smaller than that of the axion, i.e. $|\phi| \ll|\sigma|$, as can be seen by setting $\varphi_{\mathrm{RH}} \approx 0$ in (3.7). This in turn suggests that the dark matter

\footnotetext{
${ }^{12}(3.21)$ would overestimate the initial velocity if $\varphi_{\mathrm{DM}}$ at the end of inflation is just about to start oscillating, and (3.23) can also overestimate the free-streaming distance, as one sees by comparing with the exact expression (3.22). Considering these to be compensated by the factor $C \sim 10$ in (3.13) yields a naive estimate of $B \sim 1$.
} 
degree of freedom becomes dominated by the axion field, $\varphi_{\mathrm{DM}} \simeq \sigma$. We also note that we have neglected the axion's self-interactions in our analyses, which is justified if the final displacement of the axion dark matter field $\left|\varphi_{\mathrm{DM}}\right|$ is sufficiently smaller than the periodicity of the axion potential $2 \pi f$, i.e.,

$$
\left|\theta_{\star}\right| \lesssim 1
$$

If instead the field displacement exceeds half the periodicity, $\left|\varphi_{\mathrm{DM}}\right|>\pi f$, then the axion field would get trapped not in the minimum around which we have been expanding the axion potential, but in another minimum that lies near $\varphi_{\mathrm{DM}}$. The distance to this nearby minimum at the onset of the oscillation would typically be $\sim f$, hence the relic abundance in such cases is given by (2.9) with a misalignment angle $\left|\theta_{\star}\right| \sim 1$. Anharmonic corrections to the abundance computation of (2.9) would become important if the axion, after the free-streaming, lands on a point that happens to be close to a potential maximum.

We should also remark that there is no dark matter isocurvature perturbation in our inflaxion scenario, since $m_{\sigma 0}>H_{\text {inf }}$ and so inflation is effectively single-field.

\subsection{Numerical examples}

In this subsection we study the inflaton-axion dynamics in concrete examples by numerically solving the full set of equations of motion in a flat FRW universe. The coupled equations of motion of the homogeneous inflaton and axions fields that incorporate the decay of the scalar particles as effective friction terms are given in (B.8) in appendix B. The total energy-momentum tensor of the homogeneous fields can be written in the form of a perfect fluid,

$$
T_{\mu \nu}^{\sigma \phi}=\rho_{\sigma \phi} u_{\mu} u_{\nu}+p_{\sigma \phi}\left(g_{\mu \nu}+u_{\mu} u_{\nu}\right)
$$

where $u^{\mu}$ is a velocity vector normalized as $u_{\mu} u^{\mu}=-1$, with its spatial components vanishing in the Cartesian coordinates, $u^{i}=0$. The energy density and pressure of the inflaton-axion system is

$$
\begin{aligned}
& \rho_{\sigma \phi}=\frac{1}{2} \dot{\sigma}^{2}+\frac{1}{2} m_{\sigma}^{2} \sigma^{2}+\frac{1}{2} \dot{\phi}^{2}+V(\phi)+\alpha \dot{\phi} \dot{\sigma} \\
& p_{\sigma \phi}=\frac{1}{2} \dot{\sigma}^{2}-\frac{1}{2} m_{\sigma}^{2} \sigma^{2}+\frac{1}{2} \dot{\phi}^{2}-V(\phi)+\alpha \dot{\phi} \dot{\sigma} .
\end{aligned}
$$

In the numerical computation we assume all the other components of the universe to be thermalized and to form a radiation fluid. ${ }^{13}$ Hence the Friedmann equation reads

$$
3 M_{\mathrm{Pl}}^{2} H^{2}=\rho_{\sigma \phi}+\rho_{\mathrm{r}}
$$

and the continuity equation is

$$
\dot{\rho}_{\sigma \phi}+\dot{\rho}_{\mathrm{r}}+3 H\left(\rho_{\sigma \phi}+p_{\sigma \phi}\right)+4 H \rho_{\mathrm{r}}=0 .
$$

\footnotetext{
${ }^{13}$ Since the axion mass arises from a coupling with a gauge force, a derivative of such a term with the gauge field or the metric also contributes to the total energy-momentum tensor. Here we include such contributions, and also those arising from $L_{\mathrm{c}}$, into the "radiation component".
} 
During the inflationary epoch, we ignore particle decay and set the radiation density to be negligible. Moreover, by considering a low scale inflation such that $T_{\mathrm{inf}} \ll \Lambda$, the axion mass is fixed to the zero-temperature value. Hence we solve the set of equations (B.8) and (3.30) by setting $\Gamma_{ \pm}=0, \rho_{\mathrm{r}}=0, m_{\sigma}=m_{\sigma 0}$.

Then, when the cosmological expansion turns from an acceleration to a deceleration, i.e. when $\dot{H} / H^{2}=-1$, we include the decay widths into the fields' equations of motion (B.8), which are thereafter solved along with (3.30) and (3.31). The temperature dependence of the axion mass is modeled as $m_{\sigma}(T)=m_{\sigma 0} \tanh \left[\lambda(\Lambda / T)^{p}\right]$ to reproduce the asymptotic behaviors (2.1) and (2.2), and $T$ is set to the radiation temperature determined via $\rho_{\mathrm{r}}=$ $\left(\pi^{2} / 30\right) g_{*} T^{4}$.

As a toy inflaton potential that smoothly connects between an inflationary plateau with the minimum (3.5), we studied a potential of the form

$$
V(\phi)=m_{\phi 0}^{2} \mu^{2}\left(1-\frac{2}{e^{\phi / \mu}+e^{-\phi / \mu}}\right) .
$$

The inflation scale for this potential is $H_{\mathrm{inf}} \simeq m_{\phi 0} \mu / \sqrt{3} M_{\mathrm{Pl}}$ until the end of inflation, and we adopted the value $\mu=4 \times 10^{14} \mathrm{GeV}$ so that $m_{\phi 0} \approx 10^{4} H_{\text {end }}$. The axion mass was chosen as $m_{\sigma 0}=10^{-2} m_{\phi 0} \approx 10^{2} H_{\text {end }}$ with $\lambda=10^{-1}, p=6, \Lambda=10^{-1} \cdot\left(3 M_{\mathrm{Pl}}^{2} H_{\mathrm{end}}^{2}\right)^{1 / 4}$. The kinetic coupling was taken to be nonzero, but much smaller than unity, $0<|\alpha| \ll 1$. We also fixed the relativistic degrees of freedom to a constant value $g_{*}=50$ for simplicity. These parameters were chosen mainly for the purpose of reducing the computational time. The reader will have noticed that the exact values are not specified for $\alpha, m_{\phi 0}, m_{\sigma 0}$, etc.; this is because the plots we show below in terms of dimensionless quantities are independent of the exact values of such parameters. Regarding the decay channel, we considered a coupling between the inflaton and fermions as $L_{\mathrm{c}}=g_{\phi f f} \phi \bar{\psi} i \gamma^{5} \psi$, and used the expressions (B.6) for the decay widths. We have performed the computation for several different values of the dimensionless coupling $g_{\phi f f}$.

The results of the numerical computations are displayed in figure 3 , where the plots in the upper row show the evolution of the dark matter field $\varphi_{\mathrm{DM}}$ in terms of physical time (upper left) and scale factor (upper right). Here, the field value of $\varphi_{\mathrm{DM}}$ is normalized by $\alpha M_{\mathrm{Pl}} H_{\text {end }} / m_{\phi 0}$, and time $t$ is in units of $2 \pi / m_{\sigma 0}$. The end of inflation when $\dot{H} / H^{2}=-1$ is set to $t_{\text {end }}=0$ and $a_{\text {end }}=1$. Each curve is plotted with a different value for the matter coupling: $g_{\phi f f}=0.1$ (orange), 0.04 (green), 0.02 (magenta), 0.01 (pink), 0 (black dashed). The bottom left panel focuses on $g_{\phi f f}=0.1$, and shows the time evolution of $\sigma$ (blue) and $\alpha \phi$ (red), in addition to $\varphi_{\mathrm{DM}}$ (orange). The normalization of the field values and time are the same as in the upper row. The bottom right panel shows the evolution of the dark matter mass $m_{\mathrm{DM}}$, which is approximately equal to the axion mass $m_{\sigma}$, for each value of $g_{\phi f f}$. The Hubble rate $H$ is also shown as the black dot-dashed line. In this plot, the values of $m_{\mathrm{DM}}$ and $H$ are normalized by $H_{\text {end }}$.

The field evolution is, of course, independent of $g_{\phi f f}$ until the end of inflation, at which one sees that the dark matter field value is given by (3.13) with $C \approx 14$. After inflation, a larger $g_{\phi f f}$ gives a larger decay width for the reheaton, and thus the radiation temperature rises more rapidly, which in turn makes the axion mass decrease faster. To understand the 

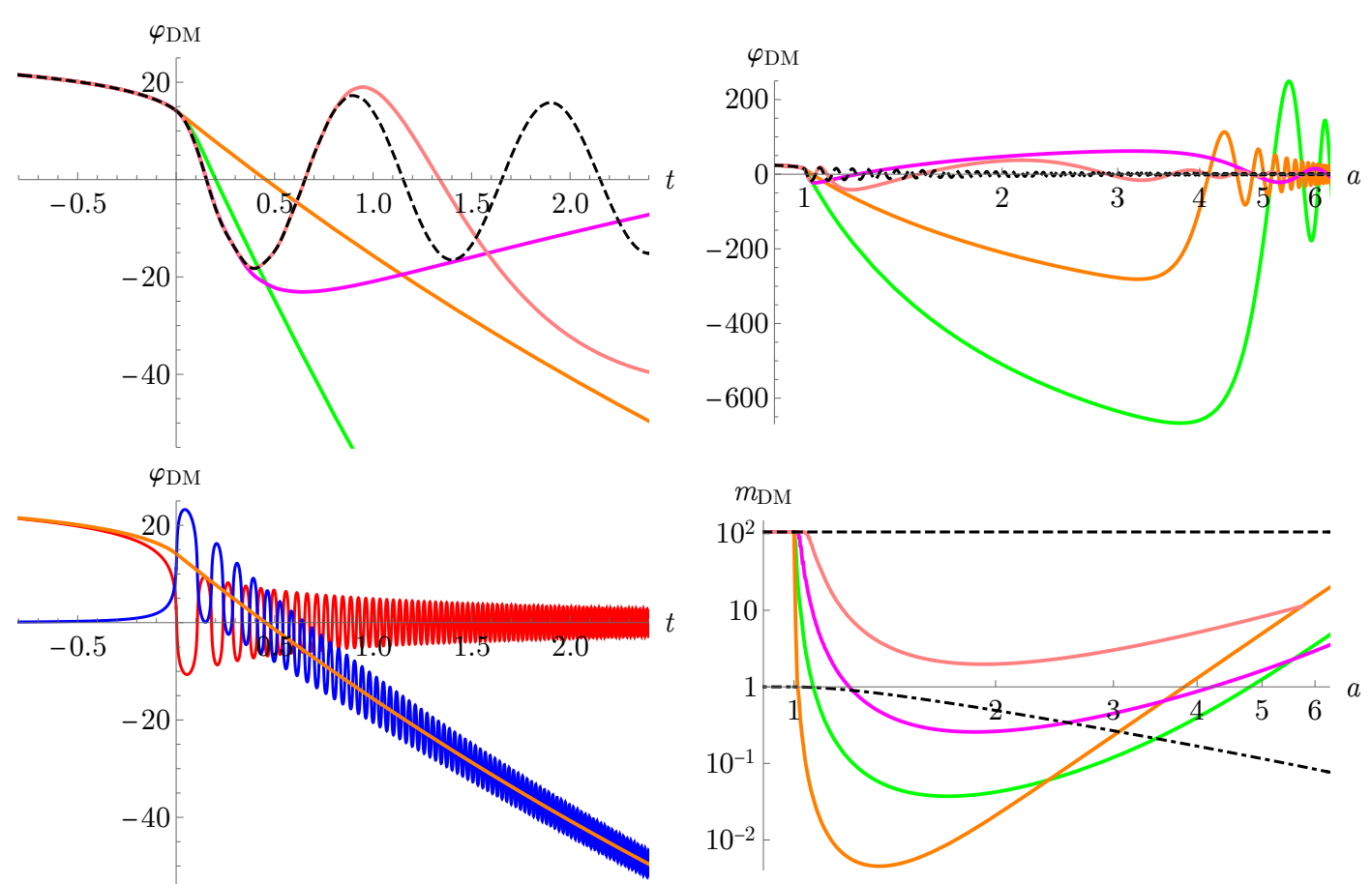

Figure 3. Time evolution of the dark matter field and its mass for a case with an inflaton mass at the vacuum $m_{\phi 0} \approx 10^{4} H_{\text {end }}$, zero-temperature axion mass $m_{\sigma 0} \approx 10^{2} H_{\text {end }}$, and strong coupling scale $\Lambda=10^{-1} \cdot\left(3 M_{\mathrm{Pl}}^{2} H_{\mathrm{end}}^{2}\right)^{1 / 4}$. The decay width of the reheaton is varied as $\Gamma_{\mathrm{RH}} / H_{\mathrm{end}} \approx 4$ (orange line), 0.7 (green), 0.2 (magenta), 0.04 (pink), and 0 (black dashed). The bottom left panel further shows $\sigma$ (blue) and $\alpha \phi$ (red), and the bottom right panel shows $H$ (black dot-dashed). The value of $\varphi_{\mathrm{DM}}$ is normalized by $\alpha M_{\mathrm{Pl}} H_{\mathrm{end}} / m_{\phi 0}$, while $m_{\mathrm{DM}}$ is normalized by $H_{\mathrm{end}}$. Time $t$ is in units of $2 \pi / m_{\sigma 0}$, and the end of inflation is set to $t_{\mathrm{end}}=0$ and $a_{\mathrm{end}}=1$. See the text for more details.

different behaviors for each value of $g_{\phi f f}$, it is instructive to see when the field evolution deviates from the case of $g_{\phi f f}=0$ (black dashed) where there is no decay and thus the dark matter field simply oscillates with frequency $m_{\sigma 0}$.

For $g_{\phi f f}=0.1$ (orange), the ratio between the reheaton's decay width and the Hubble rate at the end of inflation is $\Gamma_{\mathrm{RH}} / H_{\text {end }} \approx 4$. The elapsed time $\Delta t$ since the end of inflation until the axion mass becomes smaller than the Hubble rate is $\Delta t\left(m_{\sigma 0} / 2 \pi\right) \approx 0.2$, namely, the time scale for the dark matter field to become effectively massless is shorter than the initial oscillation period. Consequently, the field begins to free-stream with an initial velocity $\simeq \dot{\varphi}_{\mathrm{DM} \text { end. }}$. The onset of the oscillation (i.e. when (2.6) is satisfied) is at $a_{\text {osc }} \approx 3 a_{\text {end }}$, and the field displacement at this time is given by (3.24) with $B \approx 3$. This example is well described by the simple picture outlined in subsection 3.3 .

For $g_{\phi f f}=0.04$ (green), the values become $\Gamma_{\mathrm{RH}} / H_{\mathrm{end}} \approx 0.7$ and $\Delta t\left(m_{\sigma 0} / 2 \pi\right) \approx 1$. Here the dark matter field is slightly accelerated before starting to free-stream, and thus the displacement at $a_{\mathrm{osc}} \approx 4 a_{\text {end }}$ is enhanced to $B \approx 6$.

For $g_{\phi f f}=0.02$ (magenta), $\Gamma_{\mathrm{RH}} / H_{\text {end }} \approx 0.2$ and $\Delta t\left(m_{\sigma 0} / 2 \pi\right) \approx 4$. The slowlydiminishing mass drags the dark matter field for a while and forces it to free-stream towards the positive direction in the plot. However the amplitude of the initial velocity for 
the free-streaming is still $\sim\left|\dot{\varphi}_{\mathrm{DM} \text { end }}\right|$, and thus the final displacement at $a_{\mathrm{osc}} \approx 4 a_{\mathrm{end}}$ becomes $B \approx 0.5$.

For $g_{\phi f f}=0.01$ (pink), $\Gamma_{\mathrm{RH}} / H_{\text {end }} \approx 0.04$, and the mass never goes below the Hubble rate, hence the discussion in the previous subsection does not directly apply. However, due to the mass becoming sufficiently smaller than its zero-temperature value, the field dynamics is altered from the case with a constant mass.

In the bottom left plot, the higher-frequency oscillation in the inflaton and axion fields represents the reheaton degree of freedom. After the reheaton decays away, the dark matter and axion fields become approximately equivalent.

In the bottom right plot, a larger $g_{\phi f f}$ gives a smaller dark matter mass (axion mass) at the maximum temperature $T_{\max }$. After reaching its maximum value, the temperature drops as $T \propto a^{-3 / 8}$ (cf. (3.16)), and then as $T \propto a^{-1}$ after radiation domination takes over. A larger $g_{\phi f f}$ yields an earlier radiation domination, which explains the faster growth of the mass seen in the plot. The evolution of the Hubble rate also depends on $g_{\phi f f}$, however since their differences in this log plot is insignificant, we have only shown the case for $g_{\phi f f}=0.02$.

In the numerical examples presented here, all cases where the axion mass becomes smaller than the Hubble rate (i.e. $g_{\phi f f} \geq 0.02$ ) exhibit final dark matter field displacements with $B$ roughly of order unity. For these cases, the analytical arguments in section 3.3 provide a good effective description of the post-inflationary inflaxion dynamics.

\section{Parameter space}

Let us put together the conditions for the temperature-dependent inflaxion scenario. We have analyzed cases where the cosmic temperature evolves as (3.3), with the axion and inflaton masses satisfying (3.4). The resulting axion dark matter abundance is given by (2.9), with the misalignment angle (3.26). Upon deriving the abundance it was assumed that at the onset of the axion oscillation, the universe is dominated by radiation, i.e. (3.25), and that the axion mass still has not reached its zero-temperature value, i.e. (2.10). It was also assumed that the axion's self-interactions are negligible, i.e. (3.27), and the present-day cosmic temperature is below the strong coupling scale, $T_{0}<\Lambda$. The cosmic temperature at its maximum is given in (3.20), the Hubble scale when radiation domination begins in (3.18), and the temperature at the onset of the dark matter field oscillation in (2.7).

A successful cosmology with this inflaxion scenario requires the reheaton to decay and trigger radiation domination at temperatures of $T_{\text {dom }} \gtrsim 4 \mathrm{MeV}$ so as not to spoil BBN $[28,29]$, while the lifetime of the axion dark matter to be longer than the age of the universe, $\Gamma_{\mathrm{DM}}<H_{0} \approx 1 \times 10^{-33} \mathrm{eV}$. ( $\Gamma_{\mathrm{DM}}$ depends on the cosmic temperature through $m_{\sigma}(T)$, and $\Gamma_{\mathrm{DM}}<H_{0}$ should be satisfied for the zero-temperature mass $m_{\sigma 0}$. On the other hand $\Gamma_{\mathrm{RH}}$ is almost independent of $m_{\sigma}(T)$ (cf. (3.9)-(3.11)), and thus is effectively constant throughout the post-inflation epoch.) Finally, the dark matter abundance should fulfill $\Omega_{\sigma} h^{2} \approx 0.1$ to match with observations. 


\subsection{Case study: $\sigma F \tilde{F}+\phi \bar{\psi} i \gamma^{5} \psi$}

In figure 4 we show the region of the axion decay constant and inflation scale where all of the above conditions are satisfied. (Here we denote the Hubble rate until the end of inflation collectively by $H_{\text {inf }}$, considering small-field inflation models in which the time variation of the Hubble rate is tiny.) We have fixed the axion-inflaton kinetic mixing constant to $\alpha=1 / 3$, and the axion mass parameters as $\xi=10^{-1}, \lambda=10^{-1}$, and $p=4$. The parameter regions are shown for the QCD axion whose strong coupling scale is $\Lambda=200 \mathrm{MeV}$, as well as for axion-like particles that obtain masses at higher energy scales of $\Lambda=10 \mathrm{GeV}, 10^{3} \mathrm{GeV}$, $10^{5} \mathrm{GeV}$. The value of the zero-temperature axion mass is shown in the upper axes. The inflaton mass at the vacuum is fixed by the requirement of $\Omega_{\sigma} h^{2} \approx 0.1$, and is shown on the contour lines in terms of $\log _{10}\left(m_{\phi 0} / \mathrm{eV}\right)$. For the matter couplings, we considered the axion to couple to $\mathrm{SM} /$ hidden photons, and the inflaton to light fermions via

$$
L_{c}=\frac{\alpha_{\gamma}}{8 \pi f} \sigma F_{\mu \nu} \tilde{F}^{\mu \nu}+g_{\phi f f} \phi \bar{\psi} i \gamma^{5} \psi
$$

We took the dimensionless couplings as $\alpha_{\gamma}=10^{-2}$ and $g_{\phi f f}=10^{-2}$, and evaluated the decay widths as (3.9) and (3.11). Moreover, the parameter $B$ which characterizes freestreaming (cf. (3.24)) was taken as $B=1$.

The regions where the temperature-dependent inflaxion scenario allows for a successful reheating and axion dark matter generation are shown in white. On the other hand in the colored regions, the conditions listed above are violated. For the chosen set of parameters, there are four conditions that most severely constrain the parameter space: The blue region violates $m_{\sigma 0}>H_{\mathrm{inf}}$; this is the region where the conventional vacuum misalignment scenario can operate. The green region violates $m_{\sigma}\left(T_{\max }\right)<H_{\text {inf }}$ and thus lies outside the validity of the analysis in this paper. Deep inside this region the axion mass would stick to its zero-temperature value throughout the reheating process; here dark matter can instead be produced by the constant-mass inflaxion scenario as discussed in [6]. The red region violates $\left|\theta_{\star}\right|<1$; here the axion cannot account for all of dark matter without invoking anharmonic effects, as we already saw in figure 1 . Within this region, the inflaxion scenario can produce the observed dark matter abundance only if the axion field after free-streaming happens to land in the vicinity of a potential maximum. ${ }^{14}$ The orange region violates $\Gamma_{\mathrm{DM}}<H_{0}$ and thus dark matter would not survive until today. In the orange regions at small $f$ (close to the left edges of the plots) the dark matter decays predominantly through the axion-photon coupling, while in the regions at large $f$ (close to the right edges) the decay is via the inflaton-fermion coupling. The orange exclusion regions at large $f$ disappear if the fermion mass lies within $m_{\sigma 0}<2 m_{\psi}<m_{\mathrm{RH}}$ and thus the decay of dark matter into fermions is kinetically forbidden; in such a case the allowed windows extend to even larger $f$ values until they hit other conditions such as (2.10). The reheaton decays through the inflaton-fermion coupling in all four plots.

\footnotetext{
${ }^{14}$ Cases with $\left|\varphi_{\mathrm{DM}}\right|>\pi f$ which typically give $\left|\theta_{\star}\right| \sim 1$ (as discussed below (3.27)) live on the edge of the red region.
} 


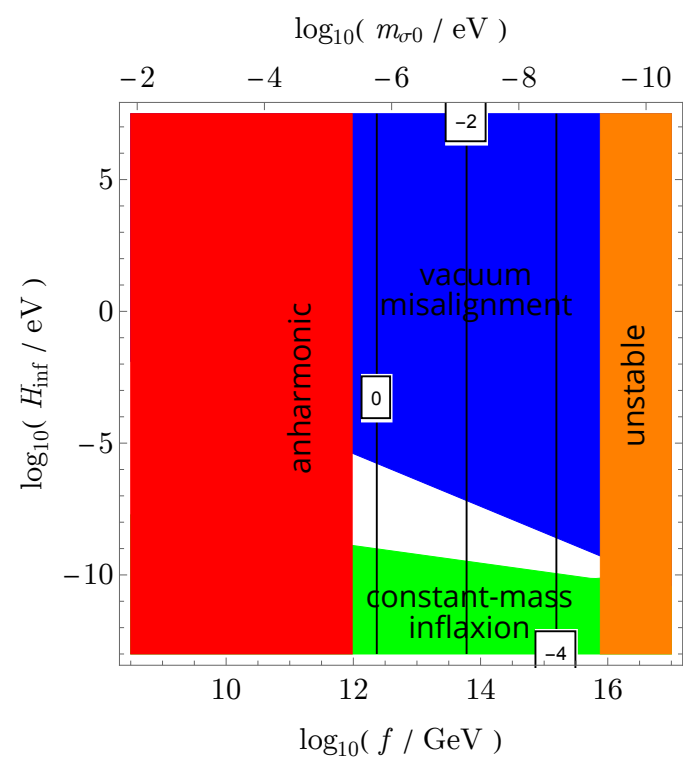

(a) $\Lambda=200 \mathrm{MeV}$ (QCD axion).

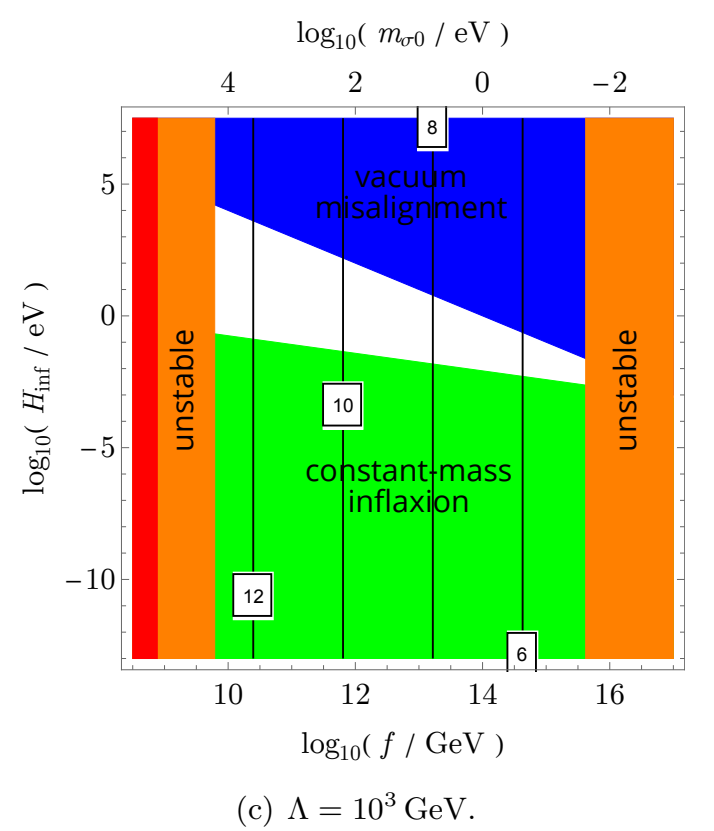

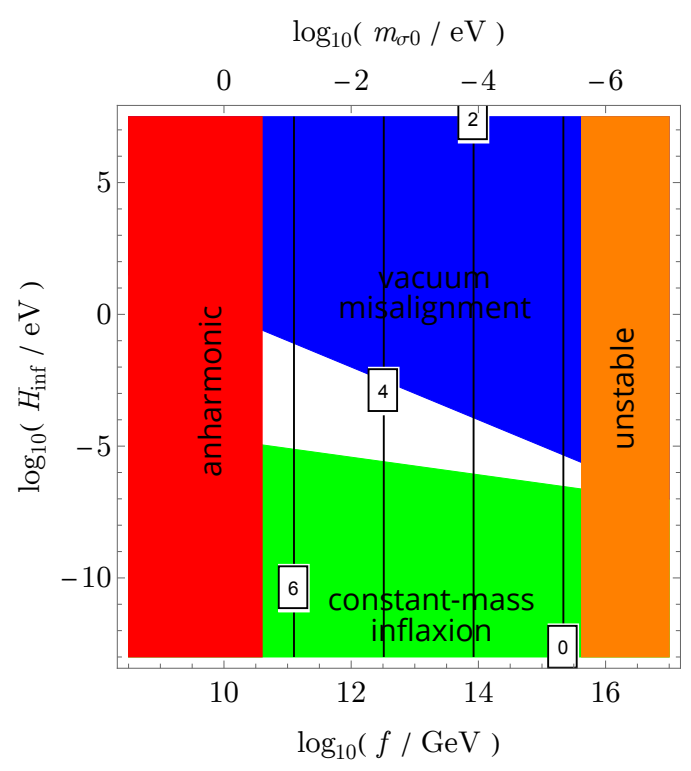

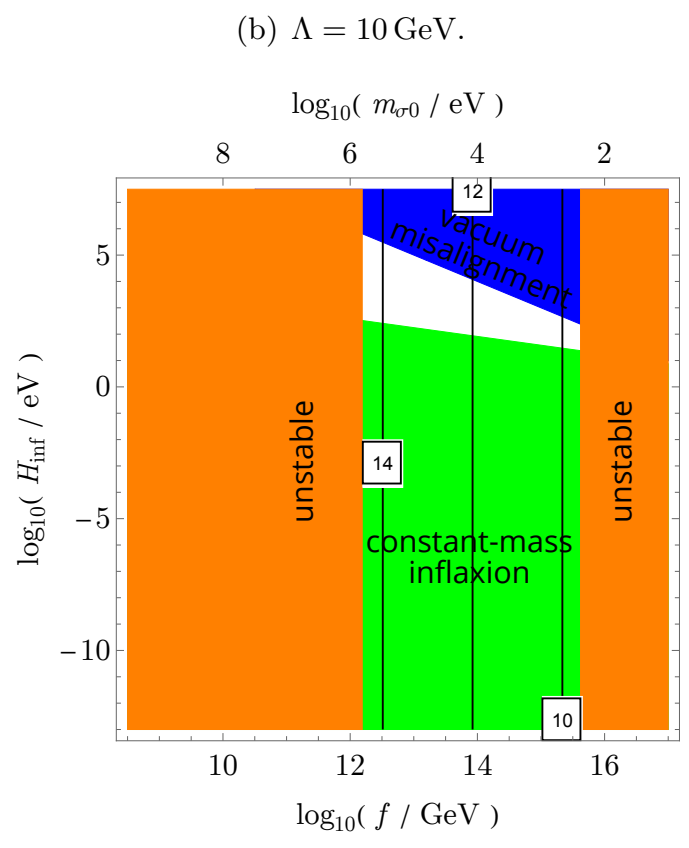

(d) $\Lambda=10^{5} \mathrm{GeV}$.

Figure 4. Parameter space for axion dark matter in the temperature-dependent inflaxion scenario, shown for the QCD axion and axion-like particles with different values of the strong coupling scale $\Lambda$. Axes are axion decay constant (bottom), zero-temperature axion mass (top), and inflationary Hubble scale (left). The allowed windows are shown in white. Colored regions lie outside the validity of our analysis since the following conditions are violated: $m_{\sigma 0}>H_{\text {inf }}$ (blue), $m_{\sigma}\left(T_{\max }\right)<H_{\text {inf }}$ (green), negligible axion self-interaction (red), and dark matter stability (orange). Contour lines show the inflaton mass at the vacuum in terms of $\log _{10}\left(m_{\phi 0} / \mathrm{eV}\right)$. The inflaton-axion mixing constant is set to $\alpha=1 / 3$, and the inflaton is coupled to fermions with $g_{\phi f f}=10^{-2}$. See the text for more details. 
We also note that for the chosen set of parameters, radiation domination takes over shortly after the end of inflation (i.e. $\Gamma_{\mathrm{RH}}>H_{\text {end }}$ ) in all of the allowed windows, except for in the vicinity of the upper right corner of the windows of figures $4(\mathrm{a})$ and $4(\mathrm{~b})$.

The inflaxion mechanism can also operate with other forms of the matter couplings, for instance, with an inflaton-photon interaction $\phi F \tilde{F}$. However with only the $\sigma F \tilde{F}$ coupling and no matter couplings for the inflaton, there is no parameter space that satisfies all the conditions, independently of the value of the coupling strength $G_{\sigma \gamma \gamma}$, if the other parameters take the same values as in the above example.

\subsection{Upper bound on inflaton mass}

Independently of the details of the matter couplings, the inflaton mass in this scenario is bounded from above as follows. For simplicity, let us here take $\kappa_{p} \sim 0.1, g_{(s) *}\left(T_{\text {osc }}\right) \sim 100$, $\lambda \sim 0.1, \xi \sim 0.1$ in the expression for the relic abundance (2.9). Then using (3.26) with $B \sim 1$ for the misalignment angle, the normalization of $\Omega_{\sigma} h^{2} \approx 0.1$ fixes the inflaton mass as

$$
m_{\phi 0} \sim 10 \mathrm{eV} \cdot|\alpha|\left(\frac{\Lambda}{200 \mathrm{MeV}}\right)^{\frac{5}{2}}\left(\frac{f}{10^{12} \mathrm{GeV}}\right)^{-\frac{3 p+5}{2 p+4}} .
$$

On the other hand, the requirement of $\left|\theta_{\star}\right|<1$ under $\Omega_{\sigma} h^{2} \approx 0.1$ bounds the decay constant as

$$
f \gtrsim 10^{12} \mathrm{GeV}\left(\frac{\Lambda}{200 \mathrm{MeV}}\right)^{-\frac{p+2}{p+3}},
$$

as shown by the red regions in the plots. Combining these expressions yields a bound on the inflaton mass that is independent of the matter coupling,

$$
m_{\phi 0} \lesssim 10 \mathrm{eV} \cdot|\alpha|\left(\frac{\Lambda}{200 \mathrm{MeV}}\right)^{\frac{4 p+10}{p+3}} .
$$

This actually sets the upper bounds on the inflaton mass in figures 4(a) and 4(b), while in figures 4(c) and 4(d) the dark matter stability condition gives stronger bounds. For axionlike particles coupled to a new strong gauge group (not QCD) with a confinement scale $\Lambda \gg 200 \mathrm{MeV}$, the upper limit (4.4) allows for a heavy enough inflaton so that perturbative reheating is easy to implement successfully.

\subsection{QCD inflaxion}

For the QCD axion, the bound (4.4) is particularly restrictive, which together with $|\alpha|<1$ gives $m_{\phi 0} \lesssim 10 \mathrm{eV}$. This rather small inflaton mass, and hence a small reheaton mass (unless $|\alpha|$ is very close to unity), poses a challenge for perturbative reheating. ${ }^{15}$ The only SM states kinematically accessible are photons and neutrinos (although not necessarily all three neutrinos, depending on the value of $m_{\phi 0}$ ). Reheating above the BBN temperature by decaying into photons requires an operator of the sort $\phi F \tilde{F}$ with an extremely large coupling strength for $m_{\phi 0} \lesssim 10 \mathrm{eV}$, such that it is largely excluded by stellar cooling

\footnotetext{
${ }^{15}$ The QCD inflaxion scenario in which the temperature of the Universe never reaches values above $\Lambda$ also has a similar issue; see the appendix in ref. [6].
} 
bounds [30]. It would be interesting to study the non-perturbative preheating phase with the photon coupling (see e.g. [31, 32]) to assess whether it is a viable option, but that is beyond the scope of the current work.

We consider then the decay into neutrinos. Gauge invariance of the SM dictates that the lowest-dimensional operator available is of dimension six:

$$
\frac{Y^{i j}}{\Lambda_{6}^{2}} \phi\left(\epsilon^{a b} \mathbf{H}_{a} \mathbf{L}_{b i}\right)\left(\epsilon^{c d} \mathbf{H}_{c} \mathbf{L}_{d j}\right)+\text { h.c. }
$$

Here $\mathbf{H}$ and $\mathbf{L}$ are the Higgs and lepton SM fields, respectively; $a, b, c, d$ are $\mathrm{SU}(2)_{L}$ indices, while $i, j$ flavor indices; $Y^{i j}$ are generalized yukawa couplings. We write this operator using two-component spinor notation, following the conventions of ref. [33]. In the scenario under consideration the electroweak symmetry is broken throughout the cosmic history (cf. figure 4(a)), and thus the operator gives rise to a yukawa coupling of the inflaton to the left-handed SM neutrinos,

$$
y_{6}^{i j} \phi \nu_{i} \nu_{j}, \quad y_{6}^{i j}=Y^{i j} \frac{v^{2}}{\Lambda_{6}^{2}},
$$

where $v$ is the electroweak scale. From here on we drop the flavor indices, for the sake of brevity, and we take the entries of $Y^{i j}$ to be of order one. The interaction (4.6) leads to a decay rate of the reheaton into neutrinos that has the form of (3.11) with $g_{\phi f f}$ replaced by $y_{6}$. The working assumption (3.25) adopted in this paper ${ }^{16}$ requires $T_{\text {dom }}$ to be above $\Lambda \approx 200 \mathrm{MeV}$, which implies

$$
y_{6}>10^{-5}, \quad \Lambda_{6}<10^{5 / 2} v \simeq 80 \mathrm{TeV},
$$

with $m_{\phi 0}=10 \mathrm{eV}$. A few comments are in order.

At the beginning of the oscillating phase the reheaton field describes a collection of non-relativistic scalar particles, which decay to produce neutrinos. These, in turn, interact among themselves via the weak force to quickly populate and thermalize the SM sector. The coupling (4.6) also implies that the scattering rate involving neutrinos and the reheaton remains faster than the Hubble expansion rate as the temperature decreases. Therefore the $\varphi_{\mathrm{RH}}$ quanta are upscattered and remain in the thermal bath with the neutrinos. This scenario, to our knowledge, has not been explored in detail yet and we leave a dedicated study of its cosmological implications to future work. ${ }^{17}$

On the particle physics side there are many constraints to take into account. First, we note that the operator (4.5) does not directly contribute to neutrino masses, given that the vacuum expectation value (VEV) of the inflaton approaches zero. Second, by replacing

\footnotetext{
${ }^{16}$ We have also considered the possibility of $T_{\text {dom }} \ll \Lambda<T_{\max }$, so that the dark matter field begins to oscillate before entering radiation domination. Here the relic abundance becomes different from the one we reviewed in section 2, and it depends also on $T_{\text {dom }}$ [19]. However we found that the parameter window for this case is tiny in our inflaxion scenario. The reason is that the following three conditions: (i) getting the observed dark matter abundance, (ii) having $H_{\text {inf }}<m_{\sigma 0}$, and (iii) $T_{\text {dom }} \ll T_{\max }$, are incompatible with each other in most of the parameter space.

${ }^{17}$ Such a case is also touched upon in the conclusions of ref. [34], and it could also have implications in addressing the $H_{0}$ tension [35].
} 
one $\mathbf{H}$ with its $\mathrm{VEV}$, it leads to the three body decay $\mathbf{H} \rightarrow \phi \nu \nu$; the corresponding width, for $\Lambda_{6}$ not too far from the upper bound (4.7), is very small and the bound from invisible Higgs decays is amply evaded. Third, we note that the operator (4.5) contains also charged leptons which, due to the $\mathrm{SU}(2)_{L}$ structure, are always accompanied by a charged Higgs, that becomes the longitudinal mode of the $W$ boson after electroweak symmetry breaking. The presence of the heavy $W$ bosons, in combination with the suppression scale of (4.7), makes it hard to probe our operator at colliders like LEP or LHC. Fourth, lepton flavor violating processes are likely to constrain some of the entries of $Y^{i j}$ in (4.5), but unlikely to exclude completely our scenario. We reserve a more detailed study of the experimental constraints for the future.

\section{Conclusions}

A kinetic mixing between the axion and the inflaton can induce axion dark matter production even if the inflationary Hubble scale is smaller than the zero-temperature axion mass. Together with our previous analysis [6], we have explored two production scenarios for axions coupled to a strong gauge group within this inflaxion framework where (1) the reheating scale is lower than the strong coupling scale and thus the axion mass stays constant throughout the cosmic history, and (2) the maximum temperature during reheating exceeds the strong coupling scale such that the axion mass temporarily vanishes. The main part of this paper was devoted to case (2), for which we found that the axion gets kicked out of the vacuum towards the end of inflation, and subsequently in the reheating epoch drifts away even further from its potential minimum. The field dynamics during reheating thus gives rise to a misalignment angle which sources axion dark matter in the later universe. This 'initial' misalignment angle is uniquely fixed by the Lagrangian parameters as (3.26), which is in contrast to the initial angle in the conventional vacuum misalignment scenario being a random variable. Our scenario further opens up new parameter space for axion dark matter, in particular the regions with low inflation scales and large axion decay constants.

Producing the QCD axion within our model requires careful consideration of the reheating process due to the small inflaton mass, $m_{\phi 0} \lesssim 10 \mathrm{eV}$, which is needed to obtain the observed dark matter abundance. We found that reheating into Standard Model neutrinos is an option, which may also yield experimentally accessible new phenomena, although we reserve a detailed study of the constraints on this scenario for the future. For axion-like particles coupled to a hidden strong gauge group that confines at an energy $\Lambda \gg 1 \mathrm{GeV}$, all the scales involved (including the inflaton mass) are higher compared to the QCD case, and thus there are many possibilities for the reheating process.

Perhaps the most exciting feature of the inflaxion framework is the inevitable link between the reheating temperature and the coupling of the axion to normal matter, which is induced by the inflaton-axion kinetic mixing. This could offer the possibility of probing the reheating scale with laboratory experiments for measuring axion couplings, and/or astrophysical experiments for constraining the dark matter lifetime. We also remark that, while this work mainly focused on the homogeneous evolution of the inflaton-axion system, 
depending on the form of the inflaton potential, inhomogeneities can develop around the end of inflation. This may give rise to axion dark matter clumps, which would further provide observational opportunities.

\section{Acknowledgments}

We thank Francesco D'Eramo, Herbi Dreiner, Juan Herrero-Garcia, Kazunori Kohri, Diego Redigolo, Andrea Romanino, and Yuko Urakawa for useful discussions.

\section{A Onset of axion oscillation}

In this appendix we analyze when an axion with a temperature-dependent mass starts to oscillate in the early universe as the mass becomes larger than the Hubble rate. Following the discussion in appendix A of [21], we explicitly solve the homogeneous Klein-Gordon equation in a flat FRW background,

$$
\ddot{\sigma}+3 H \dot{\sigma}+m_{\sigma}^{2} \sigma=0 .
$$

We consider the background universe to have a constant equation of state parameter $w$ lying within the range $-1<w<1$, so that

$$
H \propto a^{-\frac{3(1+w)}{2}} .
$$

We also assume the axion mass to have a power-law dependence on the scale factor,

$$
m_{\sigma} \propto a^{p}
$$

with $p \geq 0$. This corresponds to a temperature dependence of $m_{\sigma} \propto T^{-p}$, given that the temperature scales as $T \propto a^{-1}$. Furthermore, we assume the axion in the asymptotic past to be frozen at some field value $\sigma_{\star}$. Then the solution of (A.1) satisfying such an initial condition is obtained as

$$
\sigma=\sigma_{\star} \Gamma(\nu+1)\left(\frac{z}{2}\right)^{-\nu} J_{\nu}(z)
$$

where $J_{\nu}(z)$ is the Bessel function of the first kind, and

$$
z=\frac{2}{3(1+w)+2 p} \frac{m_{\sigma}}{H}, \quad \nu=\frac{3(1-w)}{6(1+w)+4 p} .
$$

Using this exact solution, the physical number density of the axion is computed as

$$
n_{\sigma}=\frac{1}{2 m_{\sigma}}\left(\dot{\sigma}^{2}+m_{\sigma}^{2} \sigma^{2}\right)=\frac{m_{\sigma} \sigma_{\star}^{2}}{2} \Gamma(\nu+1)^{2}\left(\frac{z}{2}\right)^{-2 \nu}\left\{J_{\nu+1}(z)^{2}+J_{\nu}(z)^{2}\right\} .
$$

In the asymptotic past $z \rightarrow 0$, the expression (A.6) takes a limiting form of

$$
n_{\sigma} \sim \frac{1}{2} m_{\sigma} \sigma_{\star}^{2},
$$



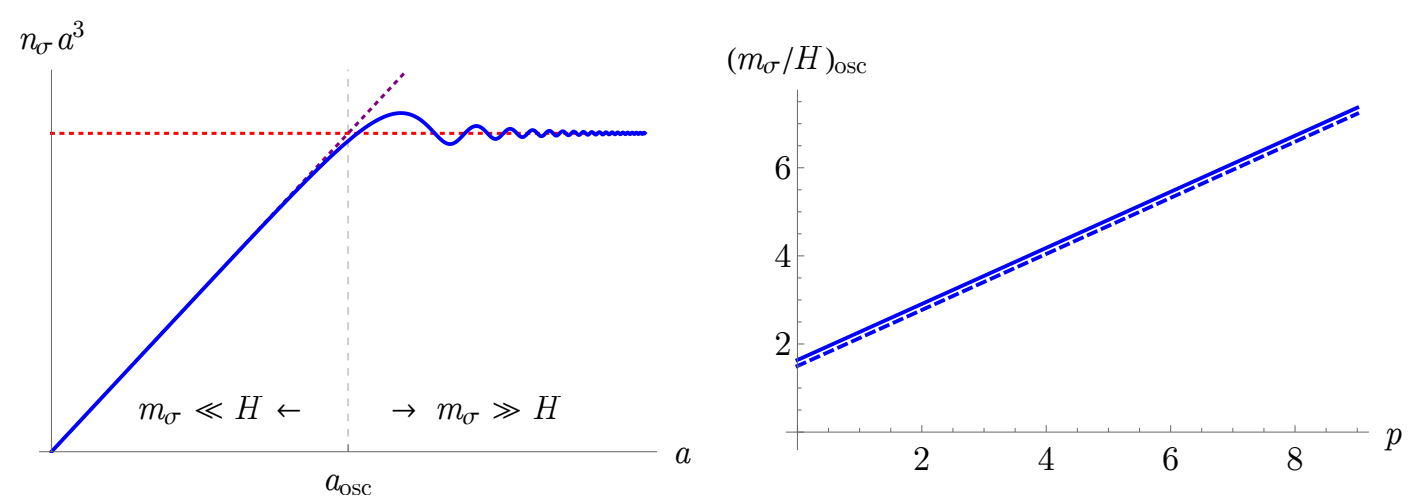

Figure 5. Left: Time evolution of the axion's comoving number density in terms of the scale factor, in $\log -\log$ scale. The dotted lines show the limiting behaviors in the asymptotic past and future. The onset of the axion oscillation is defined as when the limiting forms cross each other (see the text for details). Right: Ratio between the axion mass and the Hubble scale at the onset of the axion oscillation, as a function of the power $p$ of the temperature dependence. The equation of state of the universe is taken as $w=1 / 3$ (solid line) and $w=0$ (dashed).

as set by the initial condition. On the other hand, in the asymptotic future $z \rightarrow \infty$, it approaches

$$
n_{\sigma} \sim \frac{m_{\sigma} \sigma_{\star}^{2}}{2 \pi} \Gamma(\nu+1)^{2}\left(\frac{z}{2}\right)^{-1-2 \nu} \propto a^{-3},
$$

which manifests the conservation of the comoving number density $n_{\sigma} a^{3}$.

The comoving number density as a function of the scale factor is illustrated in the left panel of figure 5 in a log-log plot. The exact solution (A.6) is represented by the blue solid line, while the limiting forms in the asymptotic past (A.7) and future (A.8) are shown respectively as the purple and red dotted lines. Extrapolating these two limiting expressions to all times, one finds that they cross each other when the ratio between the axion mass and Hubble scale becomes

$$
\left(\frac{m_{\sigma}}{H}\right)_{\mathrm{osc}}=\frac{3(1-w)}{2 \nu}\left[\frac{\Gamma(\nu+1)^{2}}{\pi}\right]^{\frac{1}{2 \nu+1}} .
$$

We refer to this time as the 'onset' of the axion oscillation, and denote quantities at this time by the subscript (osc). This definition allows one to rewrite the conserved comoving number density in the asymptotic future in terms of quantities at the onset of the oscillation as

$$
\lim _{t \rightarrow \infty}\left(n_{\sigma} a^{3}\right)=\frac{1}{2} m_{\sigma \mathrm{osc}} \sigma_{\star}^{2} a_{\mathrm{osc}}^{3},
$$

which is convenient for computing the relic abundance as discussed in the main part of the paper.

The mass-to-Hubble ratio (A.9) at the onset of the oscillation is plotted against $p$ in the right panel of figure 5 for $w=1 / 3$ and 0 . For instance, the QCD axion possesses a temperature-dependent mass of $m_{\sigma} \propto T^{-p} \propto a^{p}$ with $p \approx 4$ at $T \gg 200 \mathrm{MeV}$; given 
that it starts to oscillate at such temperatures during the radiation-dominated epoch, ${ }^{18}$ i.e. $w=1 / 3$, the mass-to-Hubble ratio is $\left(m_{\sigma} / H\right)_{\text {osc }} \approx 4$. We also remark that for an axion-like particle whose mass depends sensitively on the temperature such that $p \gg 1$, the ratio becomes as large as $\left(m_{\sigma} / H\right)_{\text {osc }} \gg 1$. In such cases, computing the relic abundance based on a naive guess of $\left(m_{\sigma} / H\right)_{\text {osc }} \sim 1$ would lead to quite inaccurate results.

\section{B Diagonal basis}

We list expressions regarding the diagonal basis of the inflaxion Lagrangian (3.2). In this appendix we split the inflaton potential into a quadratic part and the rest as

$$
V(\phi)=\frac{1}{2} m_{\phi 0}^{2} \phi^{2}+U_{\text {int }}(\phi) .
$$

If the temperature dependence of the axion mass $m_{\sigma}$ can be ignored, the quadratic terms can be diagonalized and the Lagrangian can be rewritten as

$$
\frac{\mathcal{L}}{\sqrt{-g}}=\sum_{i= \pm}\left(-\frac{1}{2} g^{\mu \nu} \partial_{\mu} \varphi_{i} \partial_{\nu} \varphi_{i}-\frac{1}{2} m_{i}^{2} \varphi_{i}^{2}\right)-U_{\text {int }}(\phi)+L_{\mathrm{c}}[\sigma, \phi, \Psi],
$$

in terms of the diagonal fields and their masses:

$$
\varphi_{ \pm}=\left(\frac{X_{ \pm}+\beta^{2}}{X_{ \pm}^{2}+\alpha^{2} \beta^{2}}\right)^{1 / 2}\left(X_{ \pm} \sigma+\alpha \phi\right), \quad m_{ \pm}^{2}=\frac{1-X_{ \pm}}{1-\alpha^{2}} m_{\phi 0}^{2},
$$

where

$$
X_{ \pm}=\frac{1-\beta^{2} \pm \sqrt{\left(1-\beta^{2}\right)^{2}+4 \alpha^{2} \beta^{2}}}{2}, \quad \beta^{2}=\frac{m_{\sigma}^{2}}{m_{\phi 0}^{2}} .
$$

The diagonal field basis is also convenient for computing the decay widths of the scalar particles. If for instance we take the matter couplings as $(3.8)$, then by rewriting $(\phi, \sigma)$ in terms of $\left(\varphi_{+}, \varphi_{-}\right)$, the decay widths of the diagonal fields are obtained as

$$
\begin{aligned}
\Gamma\left(\varphi_{ \pm} \rightarrow \gamma \gamma\right) \simeq \frac{G_{ \pm \gamma \gamma}^{2}}{64 \pi} m_{ \pm}^{3}, \quad G_{ \pm \gamma \gamma} & =\frac{1}{X_{+}-X_{-}}\left(\frac{X_{ \pm}+\beta^{2}}{X_{ \pm}^{2}+\alpha^{2} \beta^{2}}\right)^{-1 / 2}\left(G_{\sigma \gamma \gamma}-\frac{X_{\mp}}{\alpha} G_{\phi \gamma \gamma}\right) \\
\Gamma\left(\varphi_{ \pm} \rightarrow f \bar{f}\right) & \simeq \frac{g_{ \pm f f}^{2}}{8 \pi} m_{ \pm}, \quad g_{ \pm f f}=\frac{1}{X_{+}-X_{-}}\left(\frac{X_{ \pm}+\beta^{2}}{X_{ \pm}^{2}+\alpha^{2} \beta^{2}}\right)^{-1 / 2} \frac{X_{\mp}}{\alpha} g_{\phi f f}
\end{aligned}
$$

where the fermion is assumed to be much lighter than the scalars.

Fixing the metric to a flat FRW, $d s^{2}=-d t^{2}+a(t)^{2} d \boldsymbol{x}^{2}$, the homogeneous equations of motion of the diagonal fields can be written as

$$
\ddot{\varphi}_{ \pm}+\left(3 H+\Gamma_{ \pm}\right) \dot{\varphi}_{ \pm}+m_{ \pm}^{2} \varphi_{ \pm}+U_{\text {int }}^{\prime}(\phi) \frac{\partial \phi}{\partial \varphi_{ \pm}}=0
$$

\footnotetext{
${ }^{18}$ The time variation of the relativistic degrees of freedom $g_{*}$ gives corrections to the scaling relation $T \propto a^{-1}$ as well as to the equation of state $w=1 / 3$. However as long as $g_{*}$ stays constant while the axion makes the transition from the frozen to the oscillatory phase, the discussion here is valid.
} 
where we have incorporated the decay of the scalars through $L_{\mathrm{c}}$ in the form of an effective friction term $\Gamma_{ \pm} \dot{\varphi}_{ \pm}$. These equations can be rewritten in terms of the inflaton and axion fields as

$$
\begin{gathered}
\ddot{\sigma}+\left(3 H+\frac{X_{+} \Gamma_{+}-X_{-} \Gamma_{-}}{X_{+}-X_{-}}\right) \dot{\sigma}+\alpha \frac{\Gamma_{+}-\Gamma_{-}}{X_{+}-X_{-}} \dot{\phi}+\frac{m_{\sigma}^{2} \sigma-\alpha V^{\prime}(\phi)}{1-\alpha^{2}}=0, \\
\ddot{\phi}+\left(3 H+\frac{X_{+} \Gamma_{-}-X_{-} \Gamma_{+}}{X_{+}-X_{-}}\right) \dot{\phi}+\alpha \beta^{2} \frac{\Gamma_{+}-\Gamma_{-}}{X_{+}-X_{-}} \dot{\sigma}+\frac{V^{\prime}(\phi)-\alpha m_{\sigma}^{2} \sigma}{1-\alpha^{2}}=0 .
\end{gathered}
$$

Apart from the terms involving the decay widths, these equations can also be derived directly from the original Lagrangian (3.2), and hence are exact in the limit $\Gamma_{ \pm} \rightarrow 0$, even when the axion mass depends on the temperature. The description of the scalar decay in the form of friction terms should be understood to be an effective one, which could fail for non-perturbative decay processes, or if the self-interaction $U_{\text {int }}$ is significant such that the scalar fields cannot be interpreted as a collection of particles. We also remark that, with a temperature-dependent axion mass, $\varphi_{ \pm}$do not completely diagonalize the Lagrangian; this may also yield corrections to the description of the decay in the equations of motion, as well as to the decay widths (such as those shown in (B.5) and (B.6)).

Open Access. This article is distributed under the terms of the Creative Commons Attribution License (CC-BY 4.0), which permits any use, distribution and reproduction in any medium, provided the original author(s) and source are credited.

\section{References}

[1] S. Weinberg, A New Light Boson?, Phys. Rev. Lett. 40 (1978) 223 [INSPIRE].

[2] F. Wilczek, Problem of Strong $P$ and T Invariance in the Presence of Instantons, Phys. Rev. Lett. 40 (1978) 279 [INSPIRE].

[3] R.D. Peccei and H.R. Quinn, CP Conservation in the Presence of Instantons, Phys. Rev. Lett. 38 (1977) 1440 [INSPIRE].

[4] P. Svrček and E. Witten, Axions In String Theory, JHEP 06 (2006) 051 [hep-th/0605206] [INSPIRE].

[5] A. Arvanitaki, S. Dimopoulos, S. Dubovsky, N. Kaloper and J. March-Russell, String Axiverse, Phys. Rev. D 81 (2010) 123530 [arXiv:0905.4720] [InSPIRE].

[6] T. Kobayashi and L. Ubaldi, Inflaxion Dark Matter, JHEP 08 (2019) 147 [arXiv: 1907.00984] [INSPIRE].

[7] M. Gorghetto, E. Hardy and G. Villadoro, Axions from Strings: the Attractive Solution, JHEP 07 (2018) 151 [arXiv: 1806. 04677] [INSPIRE].

[8] M. Kawasaki, T. Sekiguchi, M. Yamaguchi and J. Yokoyama, Long-term dynamics of cosmological axion strings, PTEP 2018 (2018) 091E01 [arXiv: 1806. 05566] [INSPIRE].

[9] J. Preskill, M.B. Wise and F. Wilczek, Cosmology of the Invisible Axion, Phys. Lett. B 120 (1983) 127 [INSPIRE].

[10] L.F. Abbott and P. Sikivie, A Cosmological Bound on the Invisible Axion, Phys. Lett. B 120 (1983) 133 [INSPIRE]. 
[11] M. Dine and W. Fischler, The Not So Harmless Axion, Phys. Lett. B 120 (1983) 137 [INSPIRE].

[12] K.S. Babu, S.M. Barr and D. Seckel, Axion dissipation through the mixing of Goldstone bosons, Phys. Lett. B 336 (1994) 213 [hep-ph/9406308] [INSPIRE].

[13] M. Cicoli, M. Goodsell and A. Ringwald, The type IIB string axiverse and its low-energy phenomenology, JHEP 10 (2012) 146 [arXiv:1206.0819] [INSPIRE].

[14] T. Higaki, N. Kitajima and F. Takahashi, Hidden axion dark matter decaying through mixing with QCD axion and the $3.5 \mathrm{keV}$ X-ray line, JCAP 12 (2014) 004 [arXiv:1408.3936] [inSPIRE].

[15] M. Cicoli, V.A. Diaz, V. Guidetti and M. Rummel, The $3.5 \mathrm{keV}$ Line from Stringy Axions, JHEP 10 (2017) 192 [arXiv: 1707.02987] [INSPIRE].

[16] P. Agrawal, J. Fan, M. Reece and L.-T. Wang, Experimental Targets for Photon Couplings of the QCD Axion, JHEP 02 (2018) 006 [arXiv:1709.06085] [INSPIRE].

[17] G. Lazarides, C. Panagiotakopoulos and Q. Shafi, Relaxing the Cosmological Bound on Axions, Phys. Lett. B 192 (1987) 323 [inSPIRE].

[18] T. Banks and M. Dine, The cosmology of string theoretic axions, Nucl. Phys. B 505 (1997) 445 [hep-th/9608197] [INSPIRE].

[19] G.F. Giudice, E.W. Kolb and A. Riotto, Largest temperature of the radiation era and its cosmological implications, Phys. Rev. D 64 (2001) 023508 [hep-ph/0005123] [INSPIRE].

[20] Planck collaboration, Planck 2018 results. VI. Cosmological parameters, arXiv: 1807.06209 [INSPIRE].

[21] T. Kobayashi, R. Murgia, A. De Simone, V. Iršič and M. Viel, Lyman- $\alpha$ constraints on ultralight scalar dark matter: Implications for the early and late universe, Phys. Rev. D 96 (2017) 123514 [arXiv:1708.00015] [INSPIRE].

[22] M.S. Turner, Cosmic and Local Mass Density of Invisible Axions, Phys. Rev. D 33 (1986) 889 [INSPIRE].

[23] K.J. Bae, J.-H. Huh and J.E. Kim, Update of axion CDM energy, JCAP 09 (2008) 005 [arXiv: 0806.0497] [INSPIRE].

[24] T. Kobayashi, R. Kurematsu and F. Takahashi, Isocurvature Constraints and Anharmonic Effects on QCD Axion Dark Matter, JCAP 09 (2013) 032 [arXiv:1304.0922] [INSPIRE].

[25] A.D. Linde, Axions in inflationary cosmology, Phys. Lett. B 259 (1991) 38 [InSPIRE].

[26] E.W. Kolb and M.S. Turner, The Early Universe, CRC Press, (1990).

[27] G.N. Felder, J. García-Bellido, P.B. Greene, L. Kofman, A.D. Linde and I. Tkachev, Dynamics of symmetry breaking and tachyonic preheating, Phys. Rev. Lett. 87 (2001) 011601 [hep-ph/0012142] [INSPIRE].

[28] M. Kawasaki, K. Kohri and N. Sugiyama, MeV scale reheating temperature and thermalization of neutrino background, Phys. Rev. D 62 (2000) 023506 [astro-ph/0002127] [INSPIRE].

[29] S. Hannestad, What is the lowest possible reheating temperature?, Phys. Rev. D 70 (2004) 043506 [astro-ph/0403291] [INSPIRE]. 
[30] Particle Data Group collaboration, Review of Particle Physics, Phys. Rev. D 98 (2018) 030001 [INSPIRE].

[31] P. Adshead, J.T. Giblin, T.R. Scully and E.I. Sfakianakis, Gauge-preheating and the end of axion inflation, JCAP 12 (2015) 034 [arXiv:1502.06506] [INSPIRE].

[32] J.R.C. Cuissa and D.G. Figueroa, Lattice formulation of axion inflation. Application to preheating, JCAP 06 (2019) 002 [arXiv: 1812.03132] [INSPIRE].

[33] H.K. Dreiner, H.E. Haber and S.P. Martin, Two-component spinor techniques and Feynman rules for quantum field theory and supersymmetry, Phys. Rept. 494 (2010) 1 [arXiv: 0812.1594] [INSPIRE].

[34] E. Grohs, G.M. Fuller and M. Sen, Consequences of neutrino self interactions for weak decoupling and big bang nucleosynthesis, JCAP 07 (2020) 001 [arXiv: 2002. 08557] [INSPIRE].

[35] N. Blinov, K.J. Kelly, G.Z. Krnjaic and S.D. McDermott, Constraining the Self-Interacting Neutrino Interpretation of the Hubble Tension, Phys. Rev. Lett. 123 (2019) 191102 [arXiv: 1905.02727] [INSPIRE]. 